\title{
ARTICLE OPEN \\ A built-in adjuvant-engineered mucosal vaccine against dysbiotic periodontal diseases
}

Sao Puth ${ }^{1,2}$, Seol Hee Hong ${ }^{1,3}$, Hee Sam Na ${ }^{4}$, Hye Hwa Lee ${ }^{1,2}$, Youn Suhk Lee ${ }^{1,3}$, Soo Young Kim ${ }^{1,2}$, Wenzhi Tan ${ }^{1,2}$, Hye Suk Hwang ${ }^{1,2}$, Sethupathy Sivasamy ${ }^{1,2}$, Kwangjoon Jeong ${ }^{1,2}$, Joong-Ki Kook ${ }^{5}$, Sug-Joon Ahn ${ }^{6}$, In-Chol Kang ${ }^{7}$, Je-Hwang Ryu ${ }^{3}$, Jeong Tae Koh ${ }^{3}$, Joon Haeng Rhee $e^{1,2}$ and Shee Eun Lee $e^{1,3}$

Periodontitis is associated with a dysbiotic shift in the oral microbiome. Vaccine approaches to prevent microbial shifts from healthy to diseased state in oral biofilms would provide a fundamental therapeutic strategy against periodontitis. Since dental plaque formation is a polymicrobial and multilayered process, vaccines targeting single bacterial species would have limited efficacy in clinical applications. In this study, we developed a divalent mucosal vaccine consisting of a mixture of FlaB-tFomA and Hgp44-FlaB fusion proteins targeting virulence factors of inflammophilic bacteria Fusobacterium nucleatum and Porphyromonas gingivalis, respectively. Introduction of peptide linkers between FlaB and antigen improved the stability and immunogenicity of engineered vaccine antigens. The intranasal immunization of divalent vaccine induced protective immune responses inhibiting alveolar bone loss elicited by $F$. nucleatum and $P$. gingivalis infection. The built-in flagellin adjuvant fused to protective antigens enhanced antigen-specific antibody responses and class switch recombination. The divalent vaccine antisera recognized natural forms of surface antigens and reacted with diverse clinical isolates of Fusobacterium subspecies and $P$. gingivalis. The antisera inhibited $F$. nucleatum-mediated biofilm formation, co-aggregation of $P$. gingivalis and Treponema denticola, and $P$. gingivalis-host cell interactions. Taken together, the built-in adjuvant-engineered mucosal vaccine provides a technological platform for multivalent periodontitis vaccines targeting dysbiotic microbiome.

Mucosal Immunology (2019) 12:565-579; https://doi.org/10.1038/s41385-018-0104-6

\section{INTRODUCTION}

The oral cavity is a unique ecosystem in which soft and hard tissues continuously interact with the oral microbiome, food sources and salivary secretions. Periodontitis is a highly prevalent human disease encompassing a wide range of pathologies, from mild inflammation to tooth loss. Since periodontal disease is known to be associated with the progression of various systemic diseases, ${ }^{1,2}$ prevention of periodontal disease may also affect the progression of the related systemic diseases. Dental plaque, a complex polymicrobial biofilm, is reported to be closely related with the etiology of periodontitis. ${ }^{3,4}$ Recent human microbiome studies revealed that dynamic changes in the subgingival microbiome underlie periodontitis. ${ }^{5-7}$ The disruption of microbiome homeostasis predisposes the overgrowth of diseaseassociated microbes, leading to dysbiotic periodontal inflammation. $^{8}$ Therefore, the microbial profile of the dental biofilm likely reflects the prognosis of periodontitis, and interventions targeting the disease-associated microbiome would provide a fundamental therapeutic approach for periodontitis.
Since the etiology of periodontitis has polymicrobial and multilayered characteristics, monovalent vaccines targeting single bacterial species should have limited efficacy in clinical application. An effective vaccine should prevent the shifting of the subgingival microbiome toward pathologic dysbiosis by specifically suppressing key etiologic microorganisms in the dental plaque. $^{9-11}$ Therefore, the polyvalent vaccine strategy targeting keystone periodontitis pathogens $s^{8,12}$ would be ideal for the prevention and treatment of periodontal diseases. A metagenomic analysis of the subgingival microbiome in periodontitis patients demonstrated that Porphyromonas gingivalis, one of the so-called 'red-complex bacteria', ${ }^{13}$ is significantly abundant in the diseased than resolved states. ${ }^{5} P$. gingivalis has been proposed to orchestrate the progression of periodontitis. ${ }^{12,14}$ Fusobacterium nucleatum serves a bridging colonizer, linking early colonizers with later-stage colonizers, including the red-complex species $(P$. gingivalis, Tannerella forsythia and Treponema denticola) during dental plaque formation. ${ }^{5,16} \mathrm{~F}$. nucleatum scavenges oxygen and reactive oxygen radicals in the subgingival plaque, which fosters

\footnotetext{
${ }^{1}$ Clinical Vaccine R\&D Center, Chonnam National University, Hwasun-gun, Jeonnam 58128, Republic of Korea; ${ }^{2}$ Department of Microbiology, Chonnam National University Medical School, Hwasun-gun, Jeonnam 58128, Republic of Korea; ${ }^{3}$ Department of Pharmacology and Dental Therapeutics, School of Dentistry, Chonnam National University, Gwangju 61186, Republic of Korea; ${ }^{4}$ Department of Oral Microbiology, School of Dentistry, Pusan National University, Yangsan 50612, Republic of Korea; ${ }^{5}$ Korean Collection for Oral Microbiology and Department of Oral Biochemistry, School of Dentistry, Chosun University, Gwangju 61452, Republic of Korea; ${ }^{6}$ Dental Research Institute and Department of

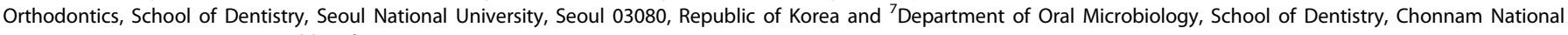
University, Gwangju 61186, Republic of Korea
}

Correspondence: Joon Haeng Rhee (jhrhee@chonnam.ac.kr) or Shee Eun Lee (selee@chonnam.ac.kr)

These authors contributed equally: Sao Puth, Seol Hee Hong

Received: 27 February 2018 Revised: 27 September 2018 Accepted: 15 October 2018

Published online: 28 November 2018 
favorable growth conditions for major strictly anaerobic periodontopathogens such as $P$. gingivalis. ${ }^{17}$ In the present study, we employ, as vaccine antigens, the Hgp44 domain polypeptide of Arg-gingipain $\mathrm{A}(\mathrm{RgpA})^{9}$ of $P$. gingivalis and an immunogenic outer membrane protein FomA of $F$. nucleatum. ${ }^{18-20}$ (Table 2) F. nucleatum is divided into subspecies (nucleatum, polymorphum, vincentii, animalis, and fusiforme) based on the polyacrylamide gel electrophoretic pattern of the whole-cell proteins and DNA homology. ${ }^{21}$ Hence a divalent vaccine targeting both $P$. gingivalis and diverse $F$. nucleatum subspecies should provide synergistic protective efficacy.

Given the oral mucosa is the primary site of periodontal inflammation, ${ }^{22}$ mucosal periodontitis vaccines inducing both systemic and mucosal immune responses ${ }^{23,24}$ would offer better protection. Mucosal secretory $\lg A(\mathrm{~S} \lg \mathrm{A})$ has potent protective efficacy at portals of entry, ${ }^{25}$ resulting in immune exclusion of microbial infections. ${ }^{26}$ We previously reported that a bacterial flagellin, Vibrio vulnificus FlaB, serves as an excellent mucosal adjuvant. $^{24,27-29}$ FomA is a major outer membrane protein of $F$. nucleatum, forming 14 transmembrane beta strands. The stable structural formation of FomA depends on the presence of supramolecular assemblies, such as micelles or bilayers. ${ }^{19}$ Therefore, the development of thermodynamically stable FomA antigens is essential for vaccination studies. ${ }^{30}$ In a previous study, we developed a truncated form of FomA (tFomA) antigen to be used as a novel subunit vaccine through a combined immunoinformatics and protein structure-based approach. ${ }^{20,30}$ Since flagellin is a proteinbased TLR agonist, we could generate recombinant proteins harboring both an antigen and built-in adjuvant FlaB by gene fusion engineering. To stimulate potent in vivo immune responses, the fusion proteins should be physico-chemically stable and retain TLR5-stimulating activity. Through the protein engineering, we come to formulate a divalent vaccine consisting of tFomA-FlaB and
Hgp44-FlaB. The built-in adjuvanted vaccine successfully induced potent immune responses in both mucosal and systemic immune compartments and was sufficiently active in preventing periodontal pathologies caused by live bacterial infections.

\section{RESULTS}

Development of FlaB-LK1-tFomA (BtA) and Hgp44-LK3-FlaB (HB) fusion proteins for a divalent periodontal vaccine targeting $F$. nucleatum and $P$. gingivalis, respectively

Periodontitis accompanies a dysbiosis composed of numerous bacterial species, and targeting all of them should not be a realist approach. Hence, we targeted two keystone pathogens, $P$. gingivalis and $F$. nucleatum, and hypothesized that their inhibition would provide significant protection against dysbiotic periodontitis. To this end, we generated several fusion proteins consisting of $\mathrm{FlaB}$ and antigens (tFomA or Hgp44) in various combinations. Since the fusion proteins contain structurally and functionally different polypeptides in a single molecule, maintaining the active structure is critical for proper in vivo function. To separate structurally stable and biologically active polypeptide domains in the fusion proteins, we developed linker peptides originated from the $\mathrm{N}$-terminal stable a-helix of Streptococcus pneumoniae PspA. ${ }^{29}$ We called the peptides LK1, LK2, and LK3, and they consisted of the PspA (2-13), PspA (2-25), and PspA (2-37) amino acids from the $\mathrm{N}$-terminus, respectively. We purified eight different proteins of the FlaB and tFomA fusion combination: FlaBtFomA (B-tA), FlaB-LK1-tFomA (B-L1-tA), FlaB-LK2-tFomA (B-L2-tA), FlaB-LK3- tFomA (B-L3-tA), tFomA-FlaB (tA-B), tFomA -LK1-FlaB (tAL1-B), tFomA-LK2-FlaB (tA -L2-B), and tFomA-LK3-FlaB (tA-L3-B). To select the optimal fusion protein, we performed sodium dodecyl sulfate-polyacrylamide gel electrophoresis (SDS-PAGE), immunoblotting and a TLR-5-dependent NFKB-reporter assay, as previously
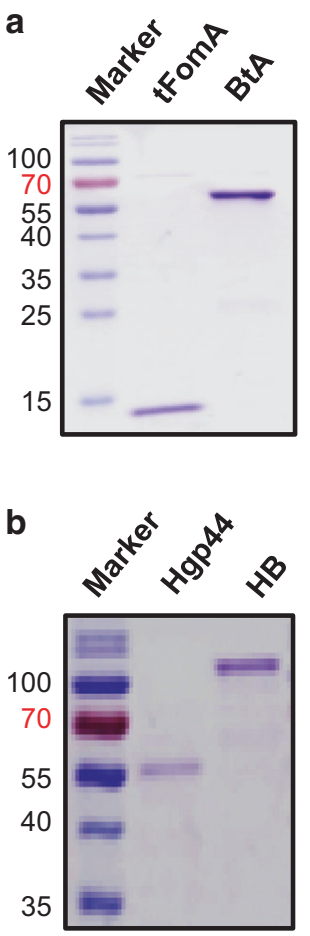

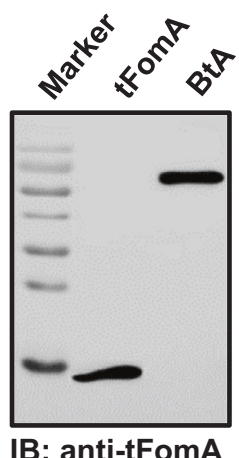

IB: anti-tFomA

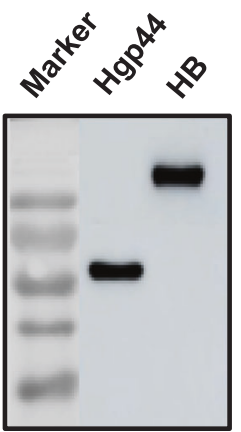

IB: anti-Hgp44

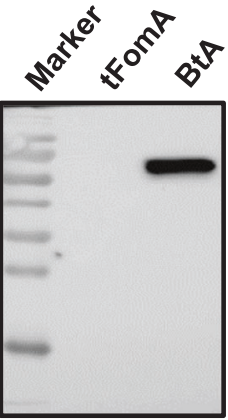

IB: anti-FlaB

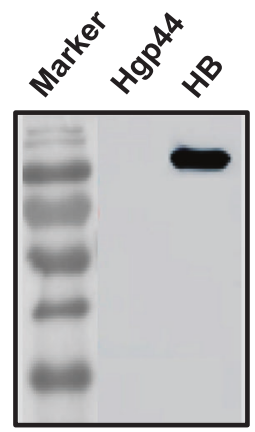

IB: anti-FlaB

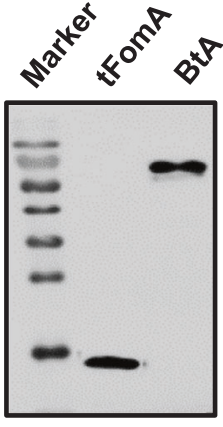

IB: anti-Fn

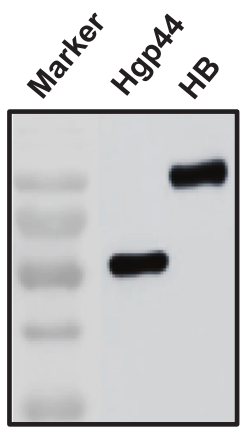

IB: anti-Pg

Fig. 1 Development of FlaB-LK1-tFomA (BtA) and Hgp44-LK3-FlaB (HB) fusion proteins for a divalent periodontal vaccine. a Characterization of a FlaB-LK1-tFomA fusion protein targeting $F$. nucleatum. Purified tFomA and FlaB-LK1-tFomA (BtA) were analyzed by sodium dodecyl sulfatepolyacrylamide gel electrophoresis (SDS-PAGE) and western blot analysis with anti-tFomA, anti-FlaB, and anti-F. nucleatum (anti-Fn) serum. b Characterization of a Hgp44-LK3-FlaB fusion protein targeting P. gingivalis. Purified Hgp44 or Hgp44-LK3-FlaB (HB) were analyzed by SDSPAGE and western blot analysis with anti-Hgp44, anti-FlaB, or anti- $P$. gingivalis (anti-Pg) serum 
described $^{27,29}$ (Supplementary Figure 1A, 1B). We also generated eight different versions of the FlaB and Hgp44 fusion: FlaB-Hgp44 (B-H), FlaB-LK1-Hgp44 (B-L1-H), FlaB-LK2-Hgp44 (B-L2-H), FlaB-LK3Hgp44 (B-L3-H), Hgp44-FlaB (H-B), Hgp44-LK1-FlaB (H-L1-B), Hgp44-LK2-FlaB (H-L2-B), and Hgp44-LK3-FlaB (H-L3-B) (Supplementary Figure 1C).

As shown in Supplementary Figures 1B, 1D, FlaB-L1-tA and HL3-B showed the strongest NFKB-stimulating activity. Of note, $\mathrm{B}-\mathrm{H}$, B-L1-H, B-L2-H, B-L3-H, and H-B did not induce TLR5-dependent NFKB activation, suggesting structural distortion in those fusion proteins. This result indicates that the TLR5-binding motif in the fusion proteins (B-H, B-L1-H, B-L2-H, B-L3-H, and H-B) was not properly exposed. We selected FlaB-L1-tA and H-L3-B as divalent vaccine components for further investigation based on the TLR5stimulating activity. We designate the B-L1-tA fusion as BtA and the H-L3-B fusion as HB. As shown in Fig. 1a, the anti-tFomA and anti- $F$. nucleatum (anti-Fn) antibody (Ab) recognized tFomA and BtA, respectively, suggesting proper antigenicity of tA. Similarly, the anti-Hgp44 and anti- $P$. gingivalis (anti- $P g$ ) Ab detected Hgp44 and $H B$, respectively (Fig. $1 \mathrm{~b}$ ). As expected, the anti-FlaB $A b$ detected the $\mathrm{FlaB}, \mathrm{Bt} A$, and $\mathrm{HB}$ proteins. These results indicate that the $\mathrm{Bt} A$ and $\mathrm{HB}$ fusion proteins are functionally active and can be used as vaccine components for the formulation of a divalent periodontitis vaccine.

Intranasal vaccination of the divalent vaccine $(B t A+H B)$ induces tFomA- and Hgp44-specific antibody responses in the serum and salivary secretions

To evaluate the antigen (Ag)-specific immune responses induced by the mucosal immunization of the divalent vaccines, we measured tFomA- or Hgp44-specific IgG and IgA titers in the sera and saliva, respectively. Mice were intranasally vaccinated three times at 2-week intervals. Two weeks after the final immunization, serum and saliva were collected, and the tFomA- or Hgp44specific $A b$ responses were assayed by ELISA. The flagellin fusion protein monovalent $(B t A)$ and divalent $(B t A+H B)$ vaccines induced significantly higher levels of anti-tFomA serum lgG $\left({ }^{*} P<0.01\right.$ for tA vs $B t A ;{ }^{* *} P<0.01$ for tA vs $\left.B t A+H B\right)$ and saliva $\lg A\left({ }^{* *} P<0.001\right.$ for tA vs $B t A ;{ }^{*} P<0.01$ for tA vs $\left.B t A+H B\right)$ than the tFom $A$ vaccine alone (Fig. 2a). Similarly, the monovalent (HB) and divalent $(\mathrm{Bt} A+\mathrm{HB})$ vaccines induced significantly higher antiHgp44-specific serum IgG $\left({ }^{* *} P<0.001\right.$ for $\mathrm{H}$ vs $\mathrm{HB} ;{ }^{* * *} P<0.001$ for $\mathrm{H}$ vs $\mathrm{BtA}+\mathrm{HB})$ and saliva IgA $\left({ }^{* * *} P<0.001\right.$ for $\mathrm{H}$ vs $\mathrm{HB} ;{ }^{* * *} P<$ 0.001 for $\mathrm{H}$ vs $\mathrm{BtA}+\mathrm{HB}$ ) responses than the $\mathrm{Hgp} 44$ vaccine alone did (Fig. 2b). These results indicated that the built-in flagellin served a potent mucosal adjuvant. The monovalent and divalent vaccine showed comparable levels of corresponding tFomA- and Hgp44-specific Ab titers. This result suggests that each hybrid vaccine (BtA or $H B$ ) did not interfere with each other's ( $H B$ or $B t A$ ) immune responses. Taken together, the divalent vaccine (BtA+ $\mathrm{HB}$ ) efficiently induced equivalent levels of tFomA- and Hgp44specific $A b$ responses compared with each monovalent vaccine (BtA or $\mathrm{HB}$ ) in both systemic and mucosal compartments.

The flagellin-antigen hybrid vaccine upregulates class switch recombination (CSR)-related genes in draining lymph nodes (LNs) To investigate whether the fusion-partner flagellin adjuvant stimulated the CSR for IgA production, we determined the germinal center (GC) B-cell responses and expression of CSRrelated genes in draining $\mathrm{LNs}$. Mice were intranasally vaccinated
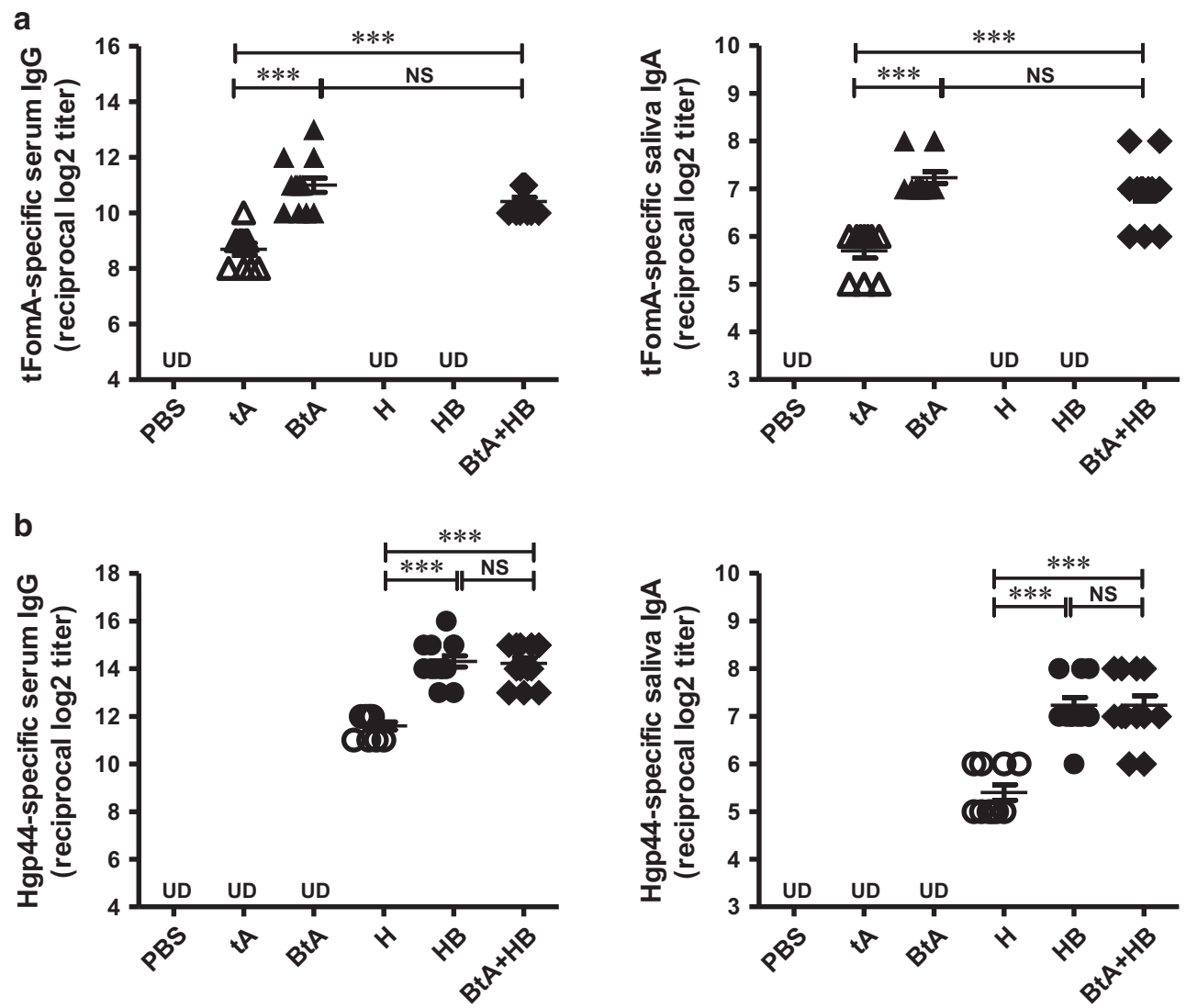

Fig. 2 An antigen-specific antibody response after intranasal vaccination. Mice were intranasally immunized with phosphate buffered saline (PBS), $1.1 \mu \mathrm{g}$ tFomA (tA), $5.1 \mu \mathrm{g}$ FlaB-LK1-tFomA (BtA), $4 \mu \mathrm{g} \mathrm{Hgp} 44(\mathrm{H}), 8 \mu \mathrm{g} \mathrm{Hgp} 44-\mathrm{LK} 3-\mathrm{FlaB}(\mathrm{HB})$ or a mixture of $5.1 \mu \mathrm{g} \mathrm{BtA}$ and $8 \mu \mathrm{g} \mathrm{HB}(\mathrm{BtA}+$ $\mathrm{HB}$ ) three times at 2-week intervals. Two weeks after the last immunization, serum or saliva were collected and tFomA- (a) or Hgp44- (b) specific serum IgG or saliva IgA titers were determined. Data are presented as the mean \pm SEM in each group. $N=10-13,{ }^{* *} P<0.01$, $* * * P<0.001$, ND not detected (under the detection limit) 


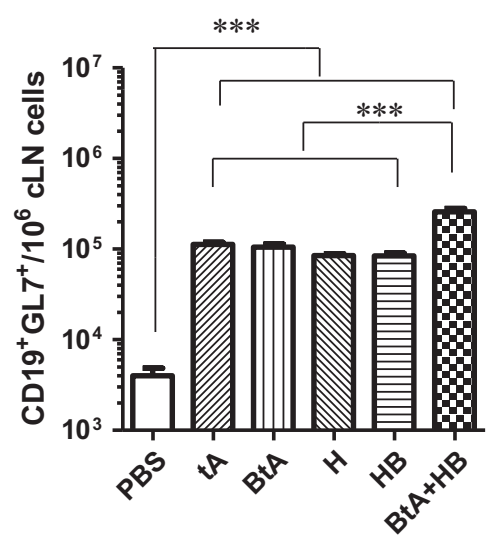

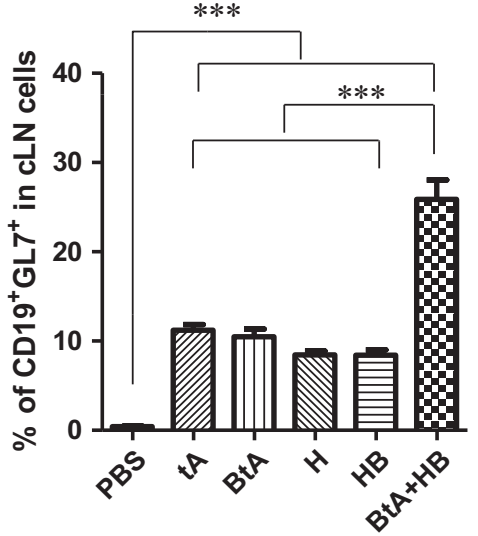

b
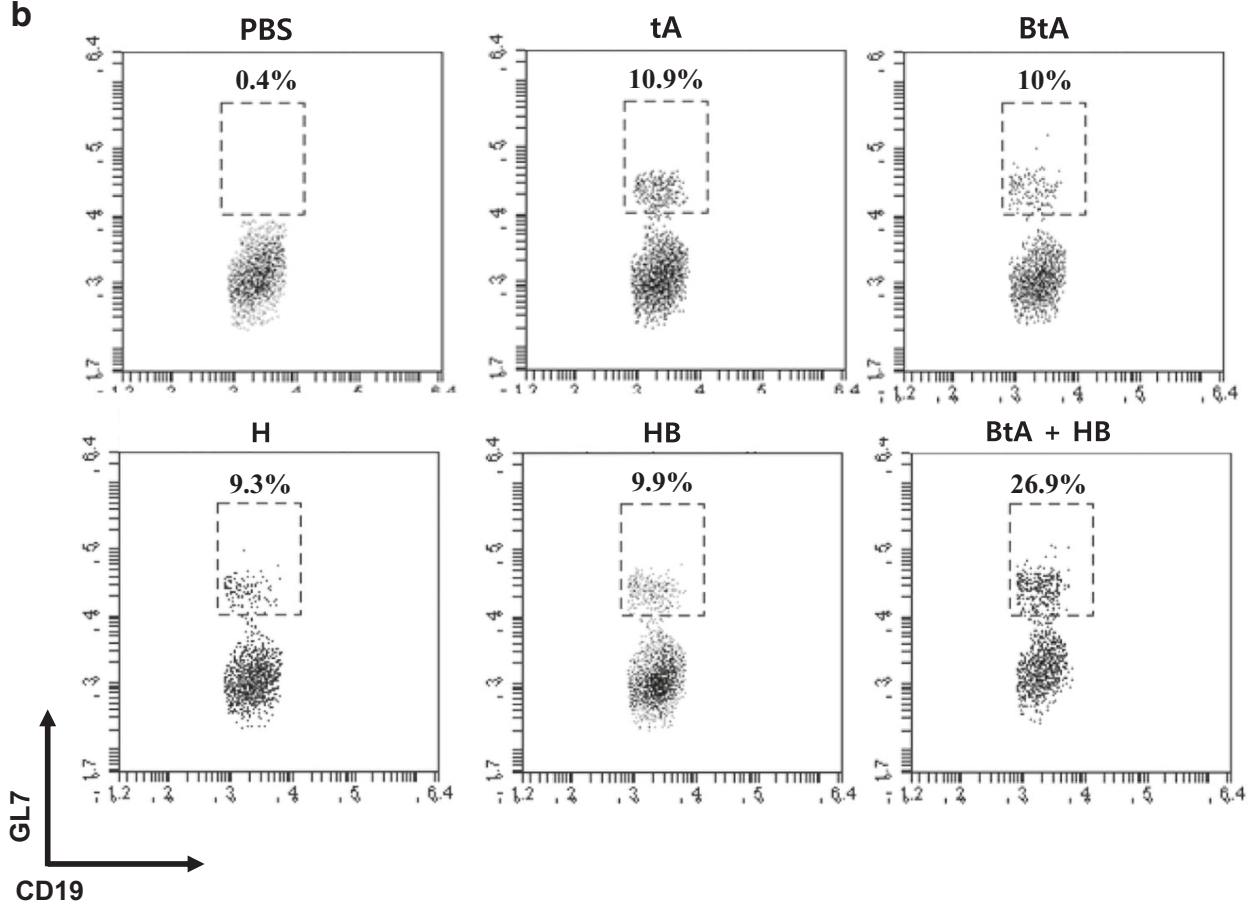

C
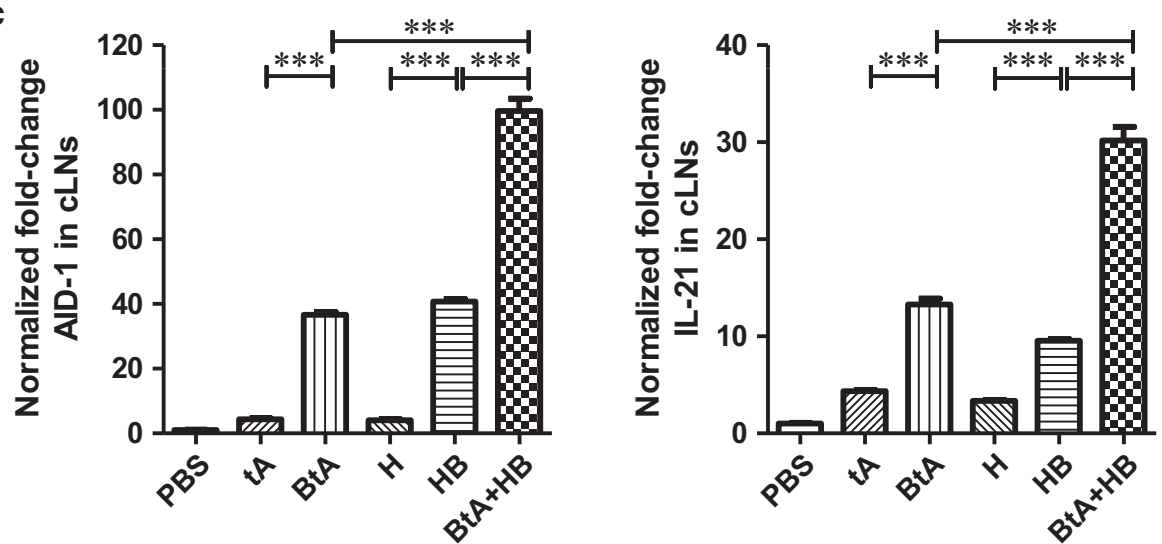

Fig. 3 Detection of GC cell populations and determination of mRNA expression levels of class switch recombination-related genes. Mice were intranasally immunized with PBS, $1.1 \mu \mathrm{g}$ tFomA (tA), 5.1 $\mu \mathrm{g} \mathrm{FlaB-LK1-tFomA} \mathrm{(BtA),} 4 \mu \mathrm{g} \mathrm{Hgp} 44(\mathrm{H}), 8 \mu \mathrm{g}$ Hgp44-LK3-FlaB (HB) or a mixture of 5.1 $\mu \mathrm{g} \mathrm{BtA}$ and $8 \mu \mathrm{g} \mathrm{HB}(\mathrm{BtA}+\mathrm{HB})$ three times at 2-week interval. Two weeks after the last immunization, the cLNs cells were prepared to evaluate GC cell populations by flow cytometry $(\mathbf{a}, \mathbf{b})$, and cytokine mRNA expression levels were determined by qRT-PCR (c). Total RNA was isolated from the $C L N$ cells, and the mRNA expression levels were measured by qRT-PCR for the indicated genes. The data are presented as the mean \pm SEM in each group. $N=5,{ }^{* *} P<0.01,{ }^{* * *} P<0.001$, NS non-significant 
a
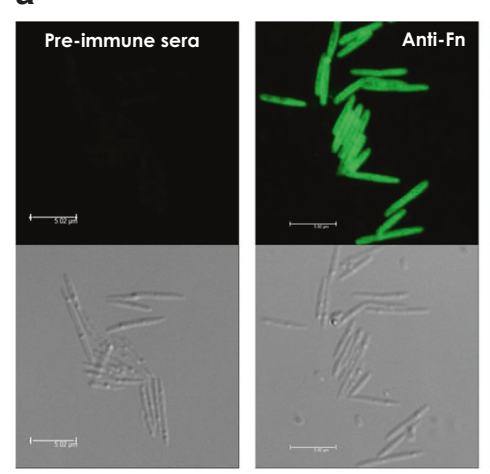

b
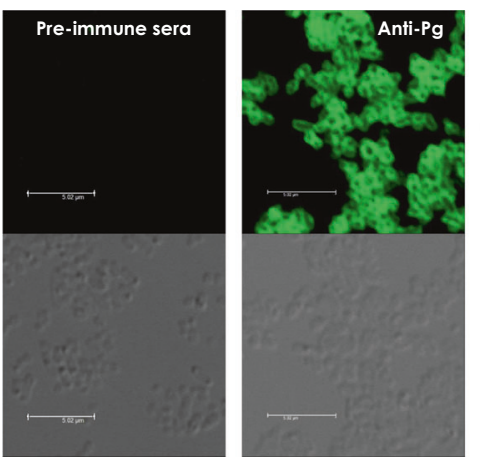

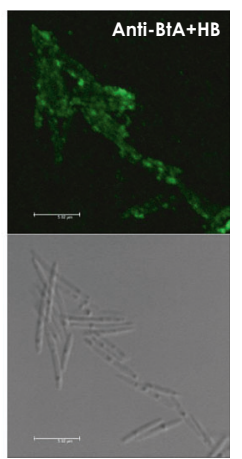

C
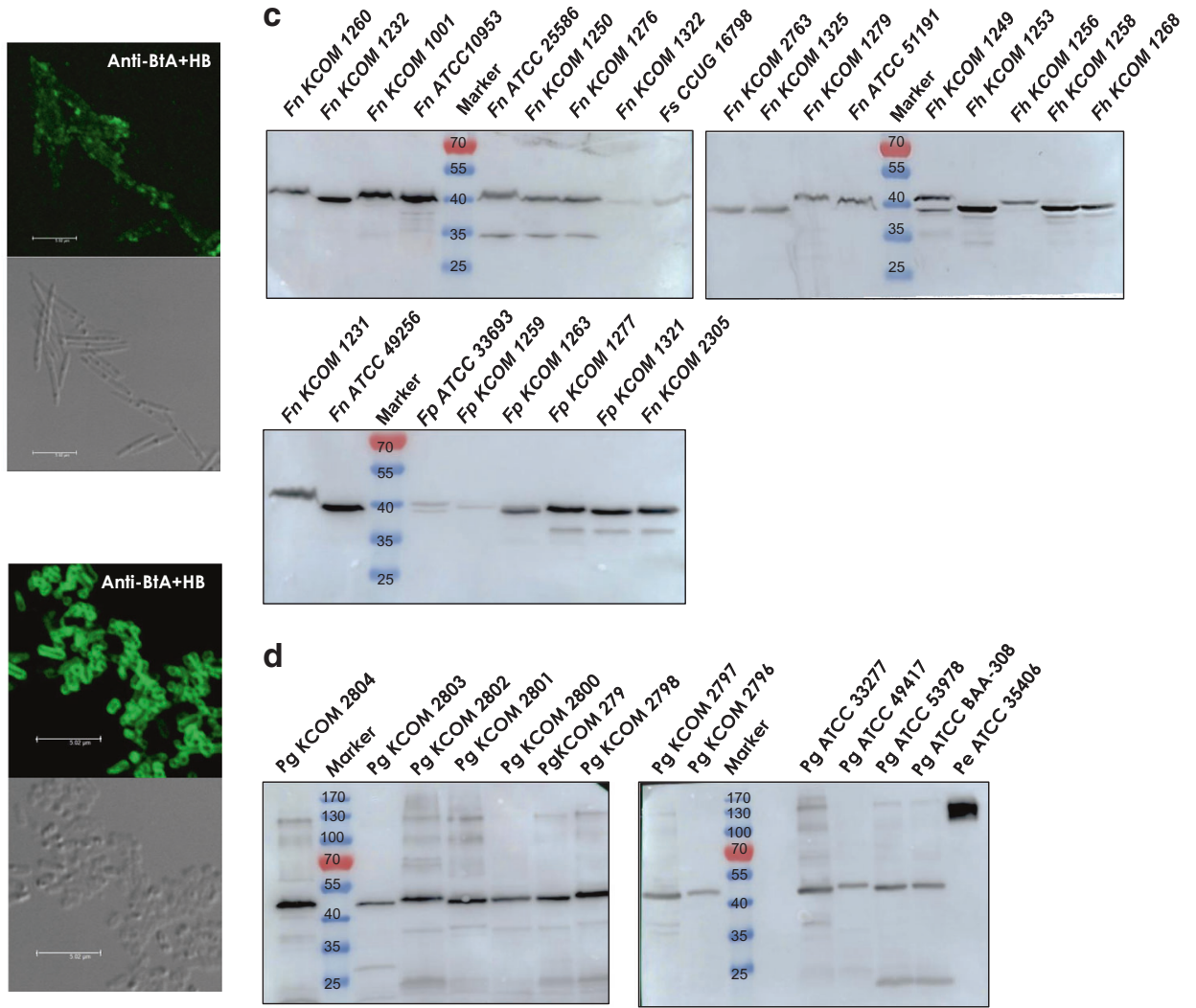

Fig. 4 Detection of cognate antigens. Immunofluorescence detection of the natural form of tFomA or Hgp44 on the surface of live $F$. nucleatum (a) or $P$. gingivalis (b), respectively. Fresh cultures of $F$. nucleatum ATCC 10953 or $P$. gingivalis ATCC 33277 were applied on the glass slides and then incubated with pre-immune, anti-Fn or anti-divalent vaccine pooled sera. The specimens were examined under confocal microscopy. Detection of specific protein band(s) by the anti-divalent sera in the clinical isolates of $F$. nucleatum or $P$. gingivalis. Bacterial lysates of $F$. nucleatum (c) or P. gingivalis (d) were analyzed by western blotting with anti-divalent sera. Fn F. nucleatum, Fs F. simiae, Fh F. hwasookii, Fp F. periodonticum, Pg P. gingivalis, Pe P. endodontalis. ATCC Americal Type Culture collection, KCOM Korean Collection for Oral Microbiology

three times at 2-week intervals. Two weeks after the final immunization, cervical lymph node (cLN) cells were collected, after which the GC B-cell population and expression of CSR-related genes, including activation-induced cytidine deaminase (AID) and IL-21, were assessed in the draining LNs. Compared with the phosphate buffered saline (PBS)-immunized mice, all the vaccinated mice ( $\mathrm{tA}, \mathrm{Bt} A, \mathrm{H}, \mathrm{HB}$, and $\mathrm{Bt} A+\mathrm{HB}$ ) showed an increased number of GC B-cells $\left(\mathrm{CD} 19^{+} \mathrm{GL} 7^{+}\right.$) in cLNs (Fig. 3a, b). The absolute number and relative composition of the GC B-cells were significantly increased in the divalent vaccine group compared to those in all other groups $(\mathrm{t} A, \mathrm{Bt} A, \mathrm{H}$, and $\mathrm{HB})$. The Tfh cell population $\left(\mathrm{CD} 4^{+} \mathrm{PD} 1^{+} \mathrm{CXCR} 5^{+}\right)$was also enhanced in the vaccinated mice (Supplementary Fig. 2). Next, we determined the mRNA expression levels of AID, an enzyme responsible for CSR, and a key IgA switching/affinity maturation cytokine IL-21. ${ }^{31}$ Notably, the mRNA expression levels of AID and IL-21 were significantly higher in the flagellin-adjuvanted monovalent vaccine (BtA or $\mathrm{HB}$ )-immunized group than those in the tFom $A$ (BtA)-immunized or Hgp44 $(\mathrm{H})$-immunized groups ( ${ }^{* * *} P<0.001$ for tB vs $B t A ;{ }^{* * *} P<0.001$ for $H$ vs $H B$ ). Strikingly, the divalent vaccine $(\mathrm{Bt} A+\mathrm{HB})$ group showed far higher enhancement of mRNA expression even compared with the already increased levels induced by flagellin-adjuvanted monovalent vaccines ( $B t A$ or $H B$ ) ( ${ }^{* *} P<0.001$ for $\mathrm{BtA}$ vs $\mathrm{BtA}+\mathrm{HB}$; ${ }^{* * *} P<0.001$ for $\mathrm{HB}$ vs $\mathrm{BtA}+\mathrm{HB}$ ) (Fig. $3 c$ ). The AID and IL21 mRNA expression levels in the divalent vaccine group $(B t A+H B)$ were similar to the sum of the monovalent vaccine (BtA and $H B$ ) mRNA expression levels. Taken together, the built-in flagellin serves a potent mucosal adjuvant by potentiating $\lg \mathrm{A}$ isotype switching, and presumably affinity maturation in the GC of draining LNs.
The divalent vaccine antisera interacts with the natural forms of the tFomA and Hgp44 antigens expressed on the surface of live $F$. nucleatum or $P$. gingivalis, respectively

To test whether the divalent vaccinated antisera can successfully recognize the natural forms of antigens (FomA and Hgp44) expressed on the bacterial cell surface, we performed immunofluorescence staining using freshly prepared $F$. nucleatum and $P$. gingivalis cells. The anti- $F n$ and anti- $P g$ sera were used as positive controls to stain surface antigens. As shown in Fig. 4, pre-immune sera could not detect any antigenic epitopes on the bacterial cell surface. As expected, anti-Fn and anti-Pg sera strongly recognized $F$. nucleatum and $P$. gingivalis surface antigens, respectively. The anti-divalent vaccine sera appeared to interact with surfaceexposed FomA (Fig. 4a) and Hgp44 (Fig. 4b). This result clearly evidences that the divalent vaccine induced functionally active, specific Abs.

The divalent vaccinated antisera detects cognate antigens in clinical isolates of $F$. nucleatum or $P$. gingivalis

To determine if the antisera raised by the divalent vaccine (BtA + $\mathrm{HB}$ ) can interact with clinical isolates of $F$. nucleatum and $P$. gingivalis, we performed an immunoblotting analysis. We separated bacterial lysates of 26 strains of $F$. nucleatum and 13 strains of $P$. gingivalis (Table 1 ) by SDS-PAGE and immunoblotted using the antisera. F. nucleatum ATCC 10953 and P. gingivalis ATCC 33277 type strains were used as positive controls. The divalent antisera detected discrete protein bands in all F. nucleatum (Fig. 4c \& Supplementary Fig. 3) and P. gingivalis clinical strains (Fig. $4 \mathrm{~d} \&$ Supplementary Fig. 3). We confirmed that the divalent antisera could detect putative FomA protein bands in all 26 human isolates 
Table 1. Bacterial strains used in this study

Bacterial strains Description Source

P. gingivalis ATCC $33277^{\top} \quad$ Type strain used for Hgp44

P. gingivalis ATCC $49417^{\top}$

P. gingivalis ATCC $53978^{\top}$

P. gingivalis ATCC BAA-308 ${ }^{\top}$

P. gingivalis KCOM 2796

P. gingivalis KCOM 2797

Human isolate

$\mathrm{KCOM}^{32}$

P. gingivalis KCOM 2798

Human isolate

$\mathrm{KCOM}^{32}$

Human isolate

$\mathrm{KCOM}^{32}$

P. gingivalis KCOM 2799

Human isolate

KCOM

P. gingivalis KCOM 2800

Human isolate

KCOM

P. gingivalis KCOM 2801

Human isolate

KCOM

P. gingivalis KCOM 2802

Human isolate

KCOM

P. gingivalis KCOM 2803

Human isolate

KCOM

P. gingivalis KCOM 2804

P. endodontalis ATCC $35406^{\top}$

Human isolate

KCOM

F. nucleatum ATCC 10953

Human isolate

F. nucleatum subsp. nucleatum ATCC

Cluster 1 (based on 16 R rRNA)

KCOM

32

Cluster 2 (based on 16R rRNA)

32

Cluster 3 (based on 16R rRNA)

32

F. nucleatum subsp. vincentii ATCC $49256^{\top}$

F. nucleatum subsp. animalis ATCC $51191^{\top}$

Cluster 4 (based on 16R rRNA)

32

F. nucleatum subsp. animalis KCOM 1001 Human isolate; Cluster 1 (based on 16R rRNA)

KCOM

F. nucleatum subsp. polymorphum

Human isolate; Cluster 3 (based on 16R rRNA)

KCOM

F. nucleatum subsp. polymorphum

Human isolate; Cluster 1 (based on 16R rRNA)

KCOM

KCOM 1232

F. nucleatum subsp. nucleatum KCOM Human isolate; Cluster 2 (based on 16R rRNA)

KCOM

F. nucleatum subsp. polymorphum KCOM 1260

Human isolate; Cluster 1 (based on 16R rRNA)

KCOM

F. nucleatum subsp. nucleatum KCOM Human isolate; Cluster 2 (based on 16R rRNA)

KCOM

1276

F. nucleatum subsp. animalis KCOM 1279 Human isolate; Cluster 4 (based on 16R rRNA)

KCOM

F. nucleatum subsp. nucleatum KCOM Human isolate; Cluster 2 (based on 16R rRNA)

KCOM 1250

F. nucleatum subsp. animalis KCOM 1325 Human isolate; Cluster 4 (based on 16R rRNA)

KCOM

F. nucleatum subsp. animalis KCOM 2763 Human isolate; Cluster 4 (based on 16R rRNA)

KCOM

F. hwasookii KCOM 1249

Human isolate; Cluster 5 (based on 16R rRNA)

KCOM

F. hwasookii KCOM 1253

F. hwasookii KCOM 1256

F. hwasookii KCOM 1258

Human isolate; Cluster 5 (based on 16R rRNA)

KCOM

Human isolate; Cluster 5 (based on 16R rRNA)

KCOM

Human isolate; Cluster 5 (based on 16R rRNA)

KCOM

F. hwasookii KCOM 1268

F. periodonticum KCOM 1259

Human isolate; Cluster 5 (based on 16R rRNA)

KCOM

Human isolate; Cluster 6 (based on 16R rRNA)

KCOM

Human isolate; Cluster 6 (based on 16R rRNA)

KCOM

Human isolate; Cluster 6 (based on 16R rRNA) KCOM

Human isolate; Cluster 6 (based on 16R rRNA) KCOM

Human isolate; Cluster 6 (based on 16R rRNA) KCOM

Human isolate; Cluster 6 (based on 16R rRNA)

32

F. periodonticum ATCC $33693^{\top}$

F. simiae CCUG $16798^{\top}$

Escherichia coli $\mathrm{DH} 5 \alpha$

E. coli ER2566

$\mathrm{F}^{-}$recA1 restriction negative

$\mathrm{F}^{-} \lambda$ fhuA2 [lon] ompT lacZ:::T7 gene1 gal sulA11 (mcrC-mrr)114::IS10 R(mcr-73:: miniTn10-TetS)2R(zgb-210::Tn10)(TetS) endA1 [dcm]

E. coli BL 21 (DE3)

hsdS gal (Aclts857 ind1 Sam7 nin5 lacUV5-T7 gene1)

Laboratory collection

New England Biolabs, Inc.

Laboratory collection

ATCC American Type Culture Collection, KCOM Korean Collection for Oral Microbiology 
of Fusobacteria (Fig. 4c) covering six clusters classified by the $16 \mathrm{~S}$ rRNA gene-based tree analysis. ${ }^{32}$ The divalent antisera also detected discrete bands in nine clinical isolates and four ATCC strains of $P$. gingivalis (Fig. $4 \mathrm{~d}$ ). In addition, the divalent antisera also recognized a $30 \mathrm{kDa}$ band in $P$. endodontalis ATCC 35406. These results suggest that the divalent vaccine $(B t A+H B)$ should be active against diverse Fusobacteria, $P$. gingivalis and $P$. endodontalis strains potentially causing periodontal diseases.

The divalent vaccine antisera and saliva inhibit $F$. nucleatum biofilm formation

F. nucleatum co-aggregates with various oral bacteria, ${ }^{32,33}$ and FomA mediates this bacterial aggregation. ${ }^{34}$ To determine whether the divalent antisera and saliva functionally inhibit $F$. nucleatum-mediated biofilm formation, we performed a colorimetric $F$. nucleatum biofilm inhibition assay as previously reported. ${ }^{\text {is }}$ Monovalent (BtA) or divalent (BtA $+\mathrm{HB}$ ) antisera inhibited $F$. nucleatum biofilm formation in a dose-dependent manner (1/4 to $1 / 32)$, while the anti-HB sera were not inhibitory even at the four-fold dilution (Fig. 5a). Notably, anti-BtA and antidivalent vaccine saliva also strongly inhibited $F$. nucleatum biofilm formation dose-dependently (1/2 to $1 / 16)$ (Fig. 5b). The biofilm inhibition effects of immune sera and saliva were also confirmed under light and confocal microscopy (Fig. 5c, d). This result suggests that antibodies in immunized sera or saliva induced by the divalent vaccines would inhibit $F$. nucleatum-mediated biofilm formation in vivo.

The divalent vaccine antisera and saliva inhibit $P$. gingivalis-host cell interactions

We also investigated the inhibitory effects of the immune sera and saliva on $P$. gingivalis-mediated hemagglutination to test whether the anti-Hgp44 vaccine could inhibit the $P$. gingivalis-host interaction during periodontitis pathogenesis. We performed a hemagglutination-inhibition assay (HIA) as previously described. ${ }^{9}$ As shown in Fig. $6 \mathrm{a}$, anti-HB and anti-BtA + HB sera inhibited $P$. gingivalis-mediated hemagglutination, while no inhibition was observed in the anti-BtA, pre-immune serum, or no serum treatments. We used the anti- $P$. gingivalis sera as a positive control for the HIA (Fig. 6a). The anti-P. gingivalis antiserum inhibited the hemagglutination up to $1: 32$ while anti-HB and anti$B t A+H B$ sera inhibited up to 1:16. For saliva, only the BtA and $\mathrm{Bt} A+\mathrm{HB}$ group inhibited hemagglutination at 1:2 (Fig. 6a). To investigate whether the vaccine antigen-specific $A b$ inhibits $P$. gingivalis infection in the oral cavity, we purified IgG from the vaccinated antisera and tested the effect on the $P$. gingivalismediated adhesion and cytotoxicity to human gingival fibroblast (HGF) cells. As shown in Fig. 6, IgG purified from the anti-BtA + HB sera significantly inhibited adhesion of $P$. gingivalis on HGFs (Fig. $6 \mathrm{~b}$ ) and $P$. gingivalis-induced LDH release from the HGFs (Fig. $6 \mathrm{c}$ ). From the HIA, adhesion and cytotoxicity assay results, we speculate that the immune sera and saliva raised by the BtA $+\mathrm{HB}$ divalent vaccine would be active in inhibiting $P$. gingivalismediated inflammation in vivo.

Immunoglobulins purified from the divalent vaccine antisera inhibit $P$. gingivalis- $T$. denticola co-aggregation

It is well documented that co-aggregation between $P$. gingivalis and Treponema denticola plays a key role in the colonization of the gingival crevice and the organization of periodontopathic biofilms. ${ }^{35}$ The Hgp44 domain of RgpA plays a critical role in coaggregation. We tested the inhibitory effect of the IgG purified from the divalent antisera on $P$. gingivalis and $T$. denticola coaggregation. As shown in Fig. $6 d$, co-aggregation of bacterial cells was observed in the $P$. gingivalis and $T$. denticola mixture $(\mathrm{Pg}+\mathrm{Td})$ and addition of $\lg G$ purified from the $\mathrm{HB}$ and $\mathrm{Bt} A+\mathrm{HB}$ antisera inhibited it.
The divalent vaccine induced protective immune responses in a $F$. nucleatum and $P$. gingivalis infection model

Next, to investigate whether the immune responses induced by the divalent mucosal vaccine would confer protection against periodontitis in vivo, we carried out a bacterial challenge experiment as previously described. ${ }^{9}$ Briefly, the vaccinated mice were orally infected with a mixture of live $F$. nucleatum and $P$. gingivalis three times (Fig. 7a). Three days after the last oral infection, we measured gene expression patterns in the gingival tissues. As shown in Fig. 7b, oral infection of $F$. nucleatum and $P$. gingivalis (NC; naive-challenged group) significantly induced IL-1 $\beta$, TNF-a, IL-6, IFN $\gamma$, and MMP9 mRNA expressions compared to the naive group (N). The vaccination group (V) showed similar gene expression patterns as the non-vaccinated naive group $(\mathrm{N})$ except for the TNFa expression. This result suggests that the vaccinespecific antibody responses in the oral cavity should have modulated inflammatory reactions. When vaccinated mice were orally challenged with the mixed bacteria, inflammation-related cytokines and MMP9 were significantly inhibited. This result clearly corroborates that the divalent vaccine effectively inhibited the inflammation induced by mixed infection with live $F$. nucleatum and $P$. gingivalis.

We evaluated whether the vaccination could prevent alveolar bone loss in the $F$. nucleatum and $P$. gingivalis-infected-mice using micro-CT analysis 40 days after the challenge. Alveolar bone loss was assessed by the bone volume density (BV/TV; bone volume/ tissue volume) value and the distance from the cemento-enamel junction to the alveolar bone crest (CEJ-ABC), as previously described. ${ }^{9}$ The mixed bacterial infection in the non-vaccinated mice (PBS) caused significant alveolar bone loss that manifested as a decreased bone volume fraction $\left({ }^{* *} P<0.01\right)$ and increased CEJ$\mathrm{ABC}\left({ }^{* * *} P<0.001\right)$ (Fig. C). Both flagellin-adjuvanted monovalent vaccines (BtA and $\mathrm{HB}$ ) protected the mice from alveolar bone loss in both bone volume density $\left({ }^{*} P<0.05\right.$ for PBS vs $B t A$; ${ }^{*} P<0.05$ for PBS vs $\mathrm{HB})$ and in CEJ-ABC $\left({ }^{* *} P<0.001\right.$ for PBS vs $B t A ;{ }^{* *} P<$ 0.001 for $P B S$ vs $H B)$. The divalent vaccine $(B t A+H B)$ also inhibited alveolar bone loss induced by mixed $F$. nucleatum and $P$. gingivalis infection, as determined by bone volume density ${ }^{* *} P<0.01$ for PBS vs $B t A+H B)$ and CEJ-ABC (***P 0.001 for PBS vs $B t A+H B)$. Notably, the divalent vaccine $(B t A+H B)$ group showed significantly higher bone volume density than the monovalent vaccine groups $\left({ }^{*} P<0.05\right.$ for $\mathrm{Bt} A+\mathrm{HB}$ vs $\mathrm{BtA} ;{ }^{*} P<0.01$ for $\mathrm{Bt} A+\mathrm{HB}$ vs $\mathrm{HB}$ ). Collectively, these results indicate that (1) mixed bacterial infection of $F$. nucleatum and $P$. gingivalis induces severe alveolar bone loss; (2) the flagellin-adjuvanted mucosal vaccine $(\mathrm{Bt} A, \mathrm{HB}$, and $\mathrm{BtA}+\mathrm{HB}$ ) targeting FomA of $F$. nucleatum and Hgp44 of $P$. gingivalis induces protective immune responses; and (3) compared to the monovalent vaccines ( $B t A$ and $H B)$, the divalent vaccine $(\mathrm{Bt} A+\mathrm{HB})$ induces superior protection. This result indicates that the divalent vaccine significantly inhibited alveolar bone loss, which is the chronic consequence of dysbiotic periodontitis. Taken together, we report here that a built-in flagellin-based divalent mucosal vaccine could serve an efficacious prophylactic measure against $F$. nucleatum-induced and $P$. gingivalis-induced dysbiotic periodontal diseases.(Fig. 7)

\section{DISCUSSION}

Periodontitis is the most prevalent human disease affecting the oral mucosa and teeth. ${ }^{2}$ For decades, enormous efforts have been made to understand the pathophysiological role of microorganisms in the development of periodontal diseases. ${ }^{3,36}$ Recent microbiome analyses have suggested that periodontitis is a dysbiotic disease elicited by the transition of the bacterial community from a healthy to a diseased state. ${ }^{7,14,37}$ In the present study, we hypothesized that a vaccine targeting both $F$. nucleatum and $P$. gingivalis could induce a synergistic intervention against the diseased dental plaque formation and subsequent 

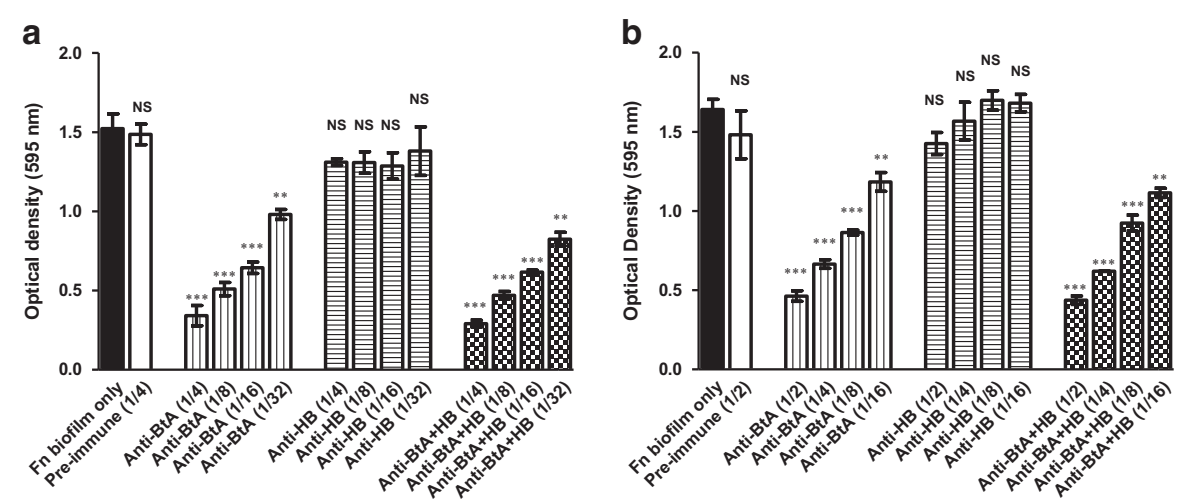

C
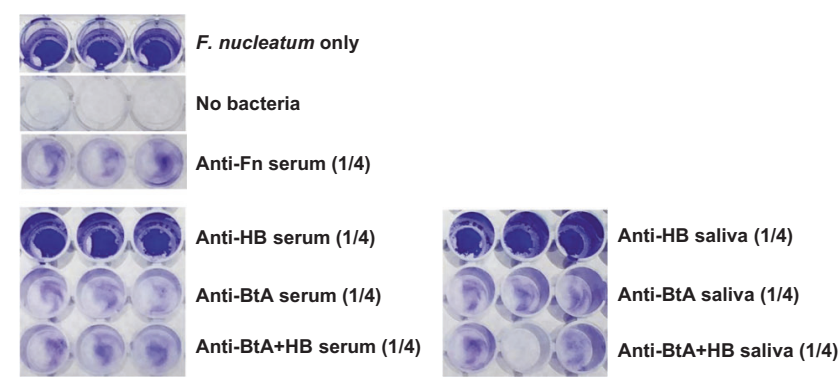

d
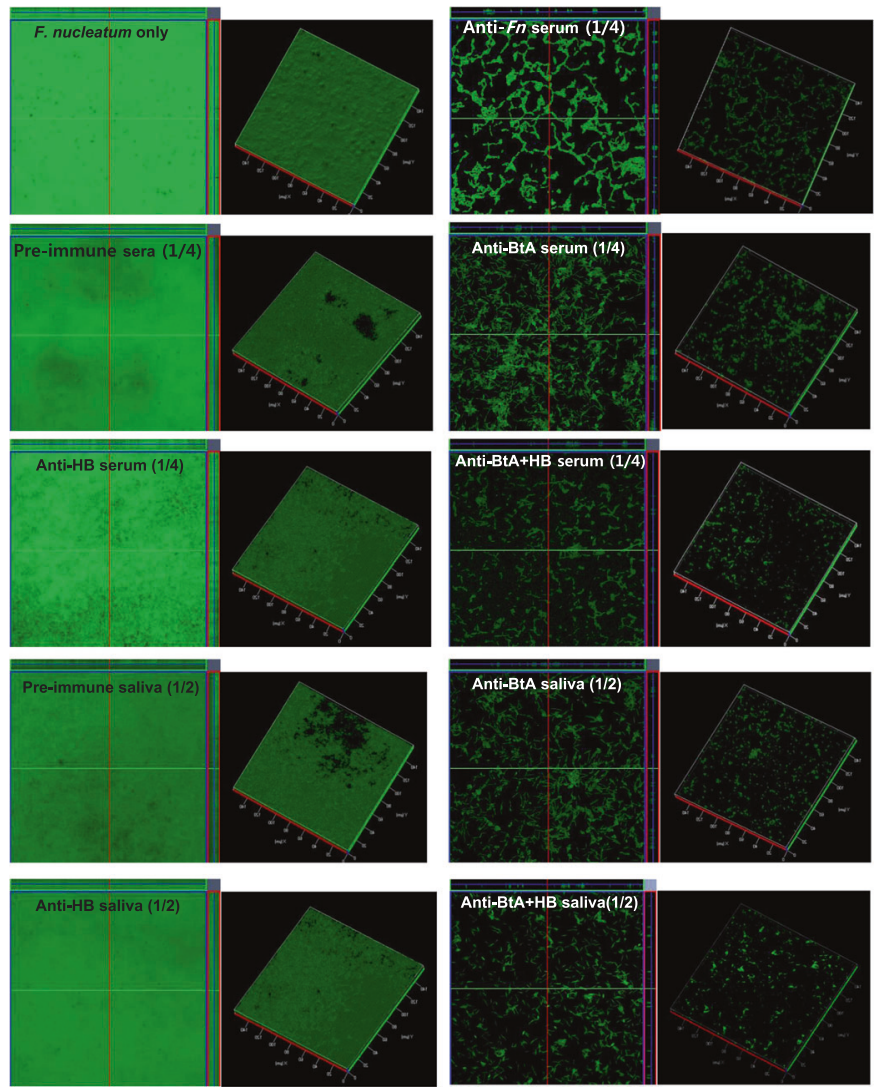

Fig. 5 Inhibition of F. nucleatum biofilm formation. The serially diluted anti-sera (a) or anti-saliva (b) were incubated with $1 \times 10^{9} \mathrm{~F}$. nucleatum ATCC 10953 cells at $37^{\circ} \mathrm{C}$ under anaerobic conditions $\left(85 \% \mathrm{~N}_{2}, 10 \% \mathrm{H}_{2}\right.$, and $\left.5 \% \mathrm{CO}_{2}\right)$ overnight. After gentle washing with PBS, the wells were stained with $25 \mu \mathrm{l}$ of $0.3 \%$ crystal violet for $15 \mathrm{~min}$ and gently washed with PBS. The stained biofilm was extracted with $100 \%$ ethanol and diluted (two-fold) with PBS to measure the absorbance at $595 \mathrm{~nm}$. The pre-immune sera or saliva was used as a control. The data are presented as the mean \pm SEM for each group. The experiments were performed with at least three replicates, ${ }^{* *} P<0.01,{ }^{* * *} P<0.001$, NS nonsignificant. c Microscopic observation of the crystal violet-stained biofilm. $\mathbf{d}$ Confocal microscopic observation of the acridine orange-stained biofilm 
a

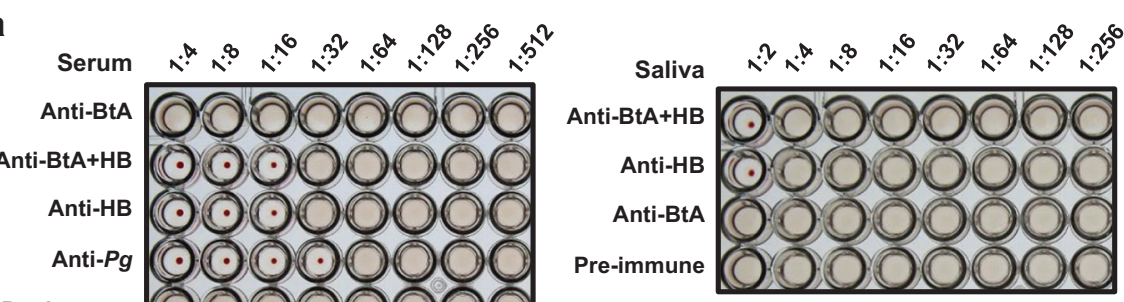

Pre-immune

No serum

b
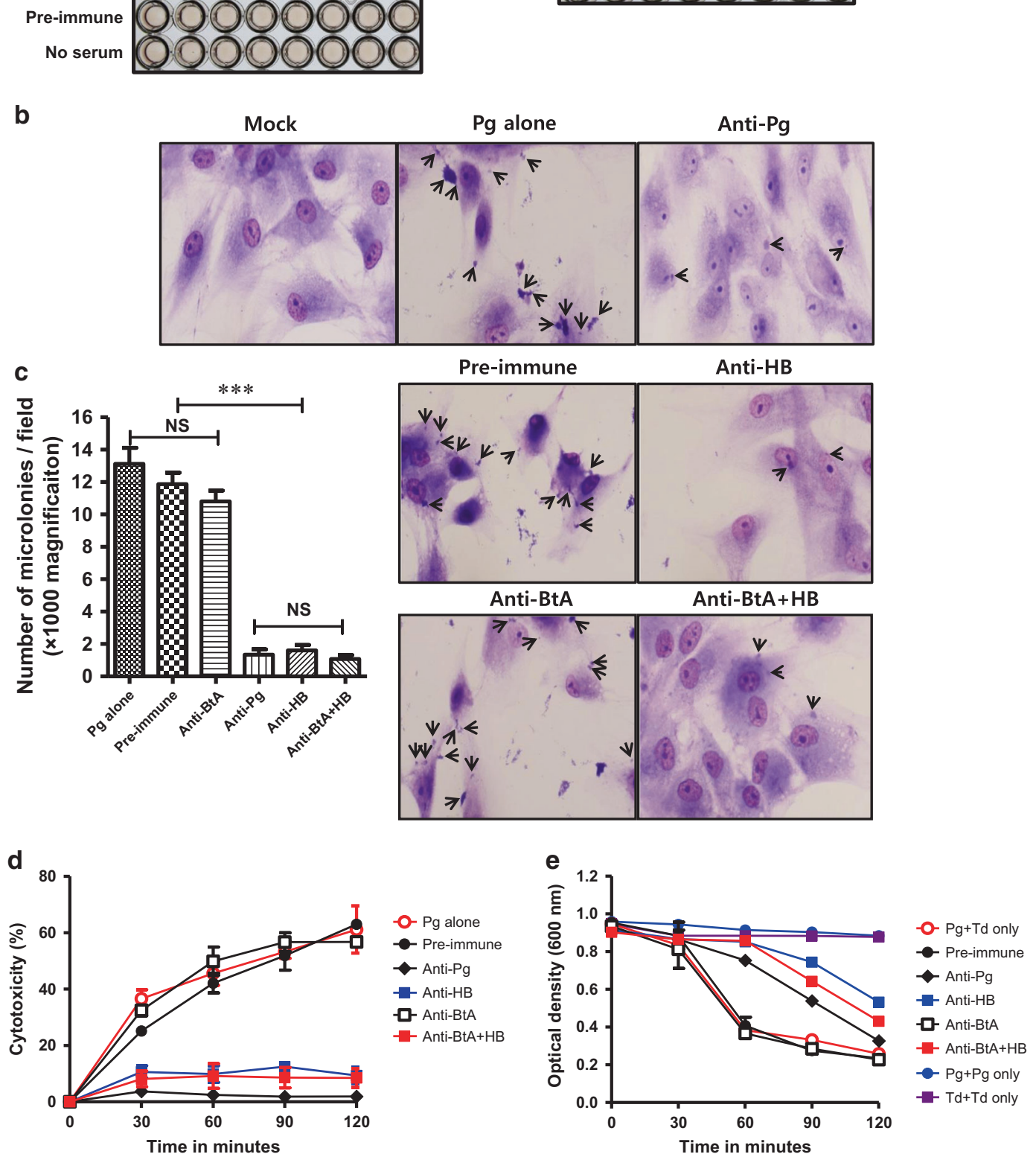

Fig. 6 Inhibition of $P$. gingivalis-host cell interactions and P.gingivalis-T. denticola co-aggregation. a Hemagglutination-inhibition assay. $P$. gingivalis was pre-incubated with the diluted anti-sera or anti-saliva induced by the periodontal vaccines and then mixed with an equal volume of mouse RBC suspension. The antisera or anti-saliva were two-fold serially diluted from $1 / 4$ to $1 / 512$ or $1 / 2$ to $1 / 256$, respectively. The anti- $P g$ sera were used as positive controls. The plate was incubated at $37^{\circ} \mathrm{C}$ for $3 \mathrm{~h}$ to observe the hemagglutination reaction. $\mathbf{b}$ Adhesion assay. Human gingival fibroblast (HGF) cells were treated with P. gingivalis ATCC 33277 at an MOI of 100 for 90 min, and the bacterial microcolonies that adhered to the HGF were counted. The numbers of microcolonies from 15 different fields were counted for each sample and expressed in graphs. c Cytotoxicity assay. HGF cells were infected with P. gingivalis ATCC 33277 at an MOI of 100 , and lactate dehydrogenase (LDH) release was measured. d Bacterial co-aggregation assay. The bacterial suspension of $P$. gingivalis ATCC 33277 was mixed with T. denticola ATCC 35405, and bacterial co-aggregation was evaluated by measuring the OD600 of the cell suspension for $2 \mathrm{~h}$ at $30 \mathrm{~min}$ intervals

periodontitis development. Here we developed a novel divalent mucosal vaccine targeting the outer membrane protein FomA of Fusobacterium nucleatum and the Hgp44 domain polypeptide of RgpA of $P$. gingivalis. Hgp44 encompasses an immunogenic region in the vicinity of the protease catalytic domain and contains a hemagglutination domain. FomA is a ubiquitous outer membrane protein in Fusobacterium spp and has been reported to function as a bacterial cell-cell adhesion molecule in dental plaque. The 
a

P. gingivalis \& F. nucleatum oral infection
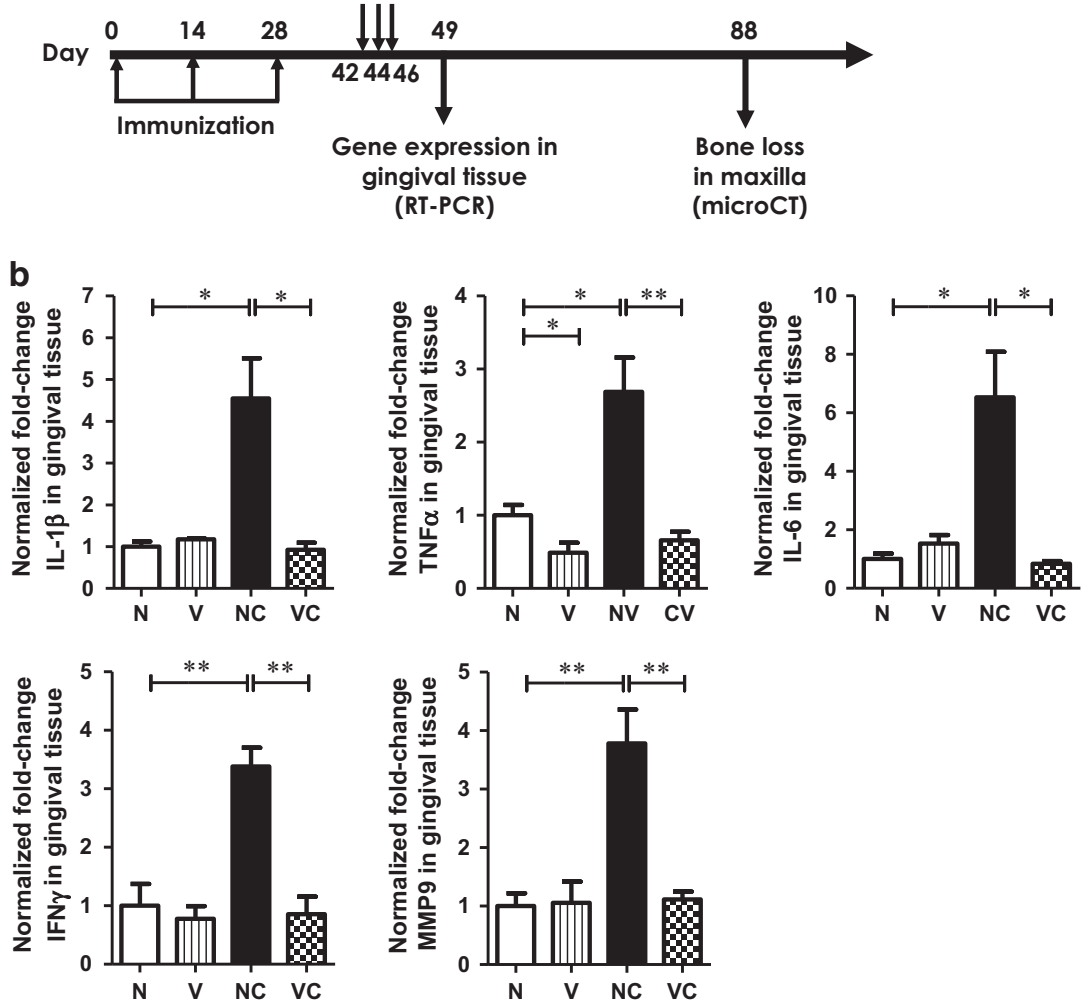

C
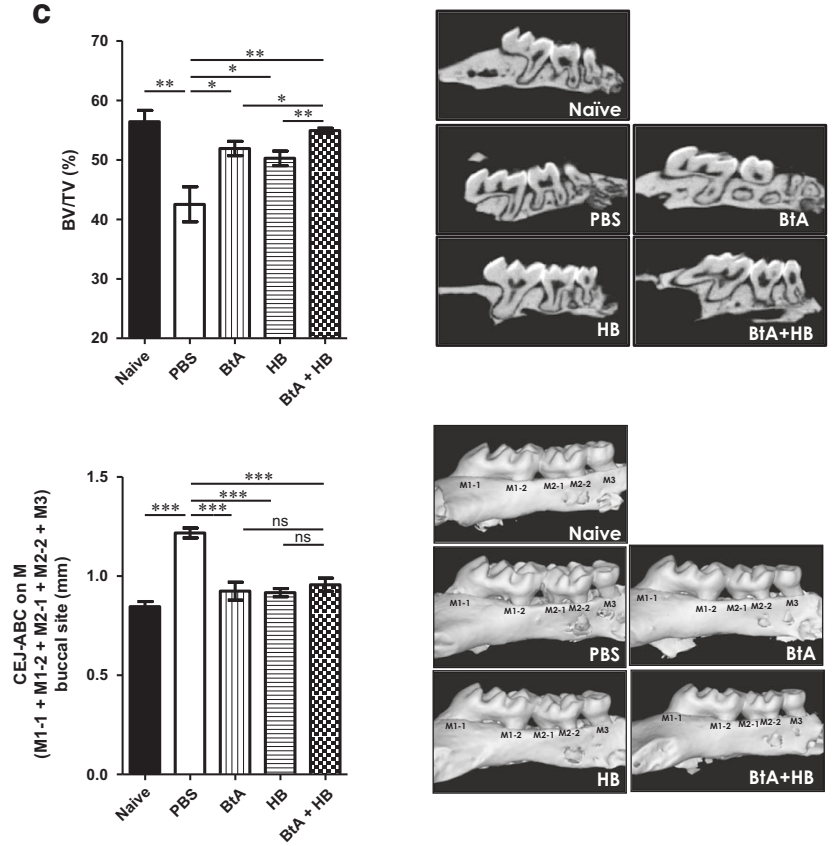

Fig. 7 The protective immune response induced by a divalent vaccine in a $F$. nucleatum and $P$. gingivalis mixed-infection model. a Experimental schedule. Mice were intranasally immunized with PBS, $5.1 \mu \mathrm{g}$ FlaB-LK1-tFomA (BtA), $8 \mu \mathrm{g} \mathrm{Hgp44-LK3-FlaB} \mathrm{(HB)} \mathrm{or} \mathrm{a} \mathrm{mixture} \mathrm{of}$ $5.1 \mu \mathrm{g} \mathrm{BtA}$ and $8 \mu \mathrm{g} \mathrm{HB}(\mathrm{BtA}+\mathrm{HB})$ three times at a two-week interval. Naive mice were used as controls. Two weeks after the final immunization, the immunized mice were orally challenged with F. nucleatum (ATCC 10953) and $P$. gingivalis (ATCC 33277 ) for 3 successive days as described in the Methods section. b Determination of inflammatory cytokines and MMP9 mRNA expressions in the gingival tissues. Three days after the final challenge, gingival tissues were prepared and cytokines and MMP9 mRNA expression levels were determined by qRT-PCR. c Determination of bone density and distance from the CEJ to the ABC. Forty days after the final challenge, alveolar bone loss was determined by measuring the bone volume density (bone volume/tissue volume; BV/TV) and distance from the CEJ to the ABC using micro-CT. The region of interest (ROI) was defined as a cuboidal bone body encompassing the roots of the first molar (M1) and second molar (M2) of the maxillae. The data are presented as the mean \pm SEM for each group. $N=8,{ }^{*} P<0.05,{ }^{*} P<0.01,{ }^{* *} P<0.001$, NS non-significant 


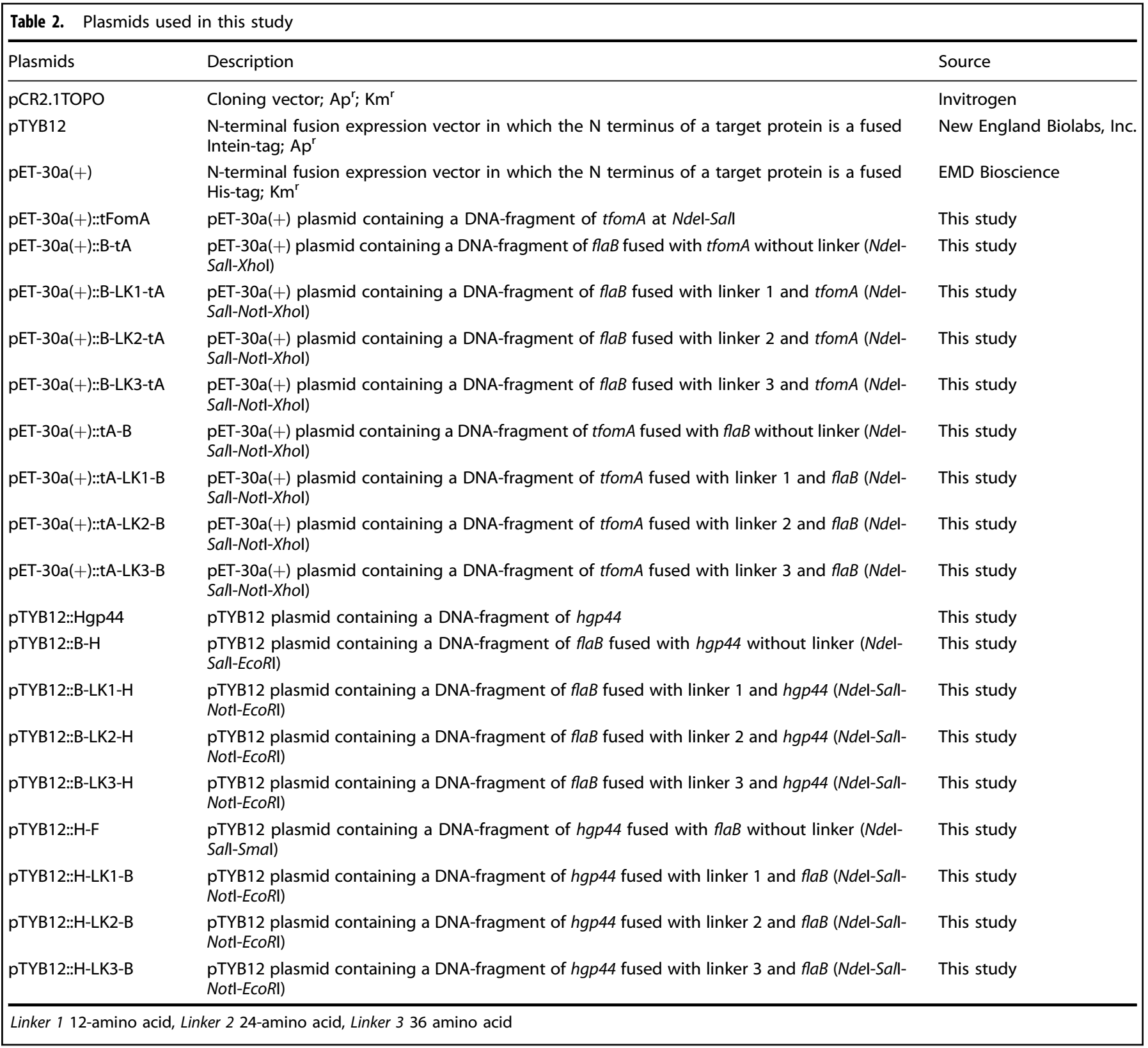

successful expression and purification of recombinant FomA protein were hard to be found in the literature, given its complicated structure having 14 transmembrane domains. To tackle this difficulty, we made a truncation of FomA (tFomA) through an in silico prediction. ${ }^{20}$ In the present study, we show that the divalent vaccine induced optimal protective immune responses effectively inhibiting alveolar bone loss elicited by $F$. nucleatum and $P$. gingivalis infection in a mouse model. The divalent vaccinated antisera successfully recognized the native FomA and Hgp44 antigenic structures on the surface of live $F$. nucleatum and $P$. gingivalis, respectively. Furthermore, the sera interacted with subspecies of Fusobacterium and $P$. gingivalis clinical isolates. We also showed that the anti-sera and anti-saliva inhibited F. nucleatum biofilm formation, P. gingivalis-T. dentiocola coaggregation and $P$. gingivalis-host interactions. This result indicates that divalent vaccines interfere not only with bacterial aggregation in the dental biofilm but also with the host-bacteria interaction on the mucosal surface. Taken together, a flagellin fusion protein-based-divalent vaccine can provide a platform for the development of multivalent periodontal vaccines. We propose the first effective protein periodontitis vaccine, to the best of our knowledge that successfully inhibits both biofilm formation and periodontal infection-mediated bone loss.

Compared with the monovalent $B t A$ or $H B$ vaccine, the divalent vaccine $(B t A+H B)$ induced equivalent levels of tFomA- and Hgp44- specific Ab responses (Fig. 2), and inhibition of $F$. nucleatum biofilm formation (Fig. 5), $P$. gingivalis- $T$. denticola coaggregation, and $P$. gingivalis-host interactions (Fig. 6). These results indicate that the individual vaccine components did not interfere with each other in antigen-specific immune responses. In addition, the divalent vaccine $(\mathrm{Bt} A+\mathrm{HB})$ showed better protection against alveolar bone loss than either of the monovalent vaccines (BtA or $\mathrm{HB}$ ) in a challenge experiment induced by a $P$. gingivalis and $F$. nucleatum mixed infection (Fig. 7). F. nucleatum bridges between early and late colonizers of the dental plaque by supporting the growth of late colonizers, including $P$. gingivalis, Aggregatibacter actinomycetemcomitans, Prevotella intermedia, Eubacterium spp., Tannerella forsythia, Selenomonas flueggei, and 
Treponema denticola. ${ }^{38,39}$ Furthermore, F. nucleatum is associated with a wide spectrum of systemic diseases, including gastrointestinal disorders, cardiovascular disease, rheumatoid arthritis, respiratory tract infections, Lemierre's syndrome, and Alzheimer's disease. ${ }^{40}$ Previous studies report that $P$. gingivalis, a member of the red-complex classified by Sokransky et al., ${ }^{41}$ is more abundant in diseased than the healthy oral tissues..$^{5-7}$ The Hgp44 domain of Arg-gingipain on the $P$. gingivalis surface directly interacts with the GPIlb/lla integrins of platelets, leading to platelet aggregation and additional atherosclerotic inflammation. ${ }^{42}$ The divalent vaccine should exert effects via two mechanisms: antibodies raised against BtA should inhibit $F$. nucleatum-mediated bacterial aggregation of microbial colonizers in the dental plaque, and antibodies raised against $\mathrm{HB}$ should modulate $P$. gingivalis-host cell interactions in the oral mucosa to down-regulate inflammatory reactions. This vaccine approach against $F$. nucleatum and $P$. gingivalis may also consequently prevent systemic diseases related to periodontal diseases.

In previous studies, we have shown that FlaB, a major component of flagellum biogenesis in $V$. vulnificus, ${ }^{43}$ is an efficacious adjuvant for various mucosal vaccines. ${ }^{9,20,27-29,44}$ Because flagellin is a proteinaceous TLR5 agonist, FlaB is an excellent partner for a fusion protein-based vaccine. To provide successful protective immune responses in the oral cavity, mucosal immunization routes should be considered for periodontal vaccines. IgA is hallmark of the mucosal immune response. ${ }^{45}$ The polymeric IgA produced by local plasma cells is transported across epithelial cells and released in luminal secretions as SIgA. The SIgA plays a crucial role in protecting the mucosal epithelium as well as in immune exclusion and anti-inflammatory actions. ${ }^{46}$ The activated $B$ cells in the GC undergo CSR, which is a critical step for long-term $A b$ responses. ${ }^{31,45,47}$ Somatic hypermutations induced by AID are crucial for CSR in the GC. ${ }^{31,45,47}$ IL-21 is the most potent cytokine that regulates B-cell functions. It directly induces B-cell CSR to promote IgA production. ${ }^{31,45,47}$ The built-in adjuvant flagellin seems to have stimulated enhanced SIgA responses in the oral cavity by increasing the GC B-cell number and activity by up-regulating follicular helper T-cells and isotype switching.

Mucosal-administered flagellin molecules eventually appear in the local draining LNs over time presumably by two routes. Nascent flagellin moves to the draining LNs through lymphatic flow or as cargo captured by TLR5 ${ }^{+} / \mathrm{CD} 11 \mathrm{c}^{+}$tissue dendritic cells (DCs). ${ }^{27}$ After intranasal immunization, the flagellin level in the cervical LN peaks after $6 \mathrm{~h}$ and is enriched in the T-cell area. ${ }^{27}$ Activated tissue DCs mobilized from the mucosal tissue mostly distribute in the T-cell area of draining LN, while free flagellin molecules that are translocated through the lymphatic system seem to be distributed rather ubiquitously. ${ }^{27,44}$ After mucosal administration of flagellin, the number of TLR5-expressing CD11 $\mathrm{c}^{+}$cells and the expression level in each cell significantly increase. ${ }^{27,44}$ Since flagellin molecules are prone to polymerize and form multimers in physiologic buffer conditions (data not shown), the flagellins that are draining to local LNs should have formed particulate structures that would be captured by subcapsular macrophages. Particulate antigens captured by subcapsular macrophages are efficiently transferred to GCS and could potentially be presented to B-cells and follicular DCs. ${ }^{48}$ These characteristics of flagellin seem to be responsible for the robust induction of the SIgA response in the mucosal compartment.

To develop an optimal subunit vaccine with a stable structure, appropriate folding of each component is essential. Since flagellin has a very stable structure and high solubility, any protein fused with flagellin would increase in stability and solubility in physiological buffer conditions. Therefore, FlaB-antigen fusion constructs had higher expression and solubility compared to antigens with poor expression. As shown in Supplementary Fig. 1, even though we successfully purified eight combinations of fusion proteins, only the Hgp44-L1-FlaB, Hgp44-L2-FlaB, and Hgp44-L3-
FlaB fusion proteins possessed TLR5-dependent NFKB-stimulating activity. In the tFomA and flagellin fusion protein combinations, FlaB-L1-tFomA showed the highest NFKB-stimulating activity. The successful expression of a flagellin-antigen hybrid protein is empirical, and an appropriate linker system seems to be an essential component for generating biologically active fusion proteins that maintain the stable structure of each component (antigen and flagellin).

As shown in Fig. 4, the pre-immune sera did not detect any components of the $F$. nucleatum or $P$. gingivalis lysates, indicating that Balb/c mice are not normally colonized by $F$. nucleatum and $P$. gingivalis. Since $F$. nucleatum and $P$. gingivalis induced severe alveolar bone loss, as determined by micro-CT analyses, the mixedinfection murine model provides an appropriate periodontitis model for vaccine study. Because the anti-divalent vaccine sera recognized both denatured $\mathrm{Ag}$ (Fig. 4c, d) and native $\mathrm{Ag}$ on the bacterial surface (Fig. 4a, b), and the anti-divalent vaccine sera successfully prevented $F$. nucleatum biofilm formation and $P$. gingivalis-mediated hemagglutination, the divalent vaccine represents a promising subunit vaccine for clinical application. We have reported that intranasal flagellin does not accumulate in olfactory nerve and bulb in our previous report. ${ }^{49}$ Based upon the same study, we have done GLP preclinical testing for safety and confirmed non-toxicity after intranasal administration and received IND approval for an intranasal influenza vaccine clinical trial. In our previous studies, we reported that TLR5 expression is well maintained in older mice and that TLR5 signaling is regulated by the upregulation of caveolin-1 in old immune cells through a direct interaction. ${ }^{28}$ This indicates that flagellin is a promising adjuvant for a vaccine for use in the elderly. Since older people have the highest rates of periodontal disease, ${ }^{50}$ flagellin should be considered as a first-line mucosal adjuvant for periodontal vaccines.

\section{METHODS}

Bacterial strains and plasmids

The bacterial strains and plasmids used in this study are listed in Table 1 and 2. Fusobacterium nucleatum subsp. polymorphum ATCC 10953 was grown in tryptic soy broth (BD, Cat. 211825) supplemented with $0.5 \%$ yeast extract (BD, REF. 212750), $0.05 \%$ cysteine (Sigma, Cat. 168149), $10 \mu \mathrm{g} / \mathrm{ml}$ of hemin (Sigma, Cat. 51280 ), $5 \mu \mathrm{g} / \mathrm{ml}$ of menadione (Sigma, Cat. M5750), $0.1 \mathrm{mM}$ of dithiothreitol (Amresco, Cat. 0281) at $37^{\circ} \mathrm{C}$ under an anaerobic condition ( $85 \% \mathrm{~N}_{2}, 10 \% \mathrm{H}_{2}$, and $5 \% \mathrm{CO}_{2}$ ). Porphyromonas gingivalis ATCC 33277 was grown as described in the previous report ${ }^{9}$.

\section{Plasmid construction and protein production}

The pTYB12 plasmid containing a DNA fragment of truncatedFomA (tFomA) at Ndel-Sall of multiple cloning sites was constructed by PCR-amplified from the genomic DNA of Fusobacterium nucleatum subsp. polymorphum ATCC 10953 using the following PCR primers: forward 5'-CATATGGTGGAGAAGAAGAACCCGAAAGATG-3' and reverse 5'-GTCGAC CAAGCTIT TTCGCCGTCATTC-3'. The Hgp44 and its fusion proteins were prepared as previously described ${ }^{9}$. In order to optimize the purification of recombinant fusion proteins, linker-peptides originated from PspA of Streptococcus pneumoniae ${ }^{29}$ were introduced between antigens (tFomA or Hgp44) and FlaB. The tFomA- or Hgp44-specific DNA fragment was separately cloned and excised from pCR2.1 TOPO vector (Invitrogen, 45-0641) with the appropriate restriction enzymes and subcloned at the $\mathrm{N}$-terminus or C-terminus of the FlaB-linker-specific DNA sequences in the $\mathrm{pET} 30 \mathrm{a}+$ or pTYB12 vectors. The DNA sequences of the resulting expression vectors were confirmed by the dideoxy-chain termination sequencing method via the Macrogen Online Sequencing Order System (http://dna. macrogen.com/kor/). As the results, the constructed plasmids were listed in Table 2. 
Anti- $F$. nucleatum and anti-P. gingivalis antibody production $F$. nucleatum antibody was generated following the previous report ${ }^{9}$. Briefly, $2 \times 10^{9} \mathrm{~F}$. nucleatum subsp. polymorphum ATCC 10953 was inactivated by treatment with $0.3 \%(\mathrm{v} / \mathrm{v})$ formalin (T\&l, BPP-9004) overnight. The inactivated $F$. nucleatum was thoroughly washed with phosphate buffered saline (PBS) and mixed with complete Freund's adjuvant (CFA) (Sigma, CAS9007-81-2) for vaccination. Six-week-old BALB/c mice were immunized with the inactivated $F$. nucleatum three times with one-week intervals by subcutaneous injection. The anti- $P$. gingivalis was produced as described in the previous report ${ }^{9}$.

Vaccination schedule

Eight-week-old female $B A L B / c$ mice were intranasally immunized with $1.1 \mu \mathrm{g}$ of tFomA (tA), $5.1 \mu \mathrm{g}$ of FlaB-tFomA (BtA), $4 \mu \mathrm{g}$ of $\mathrm{Hgp} 44(\mathrm{H}), 8 \mu \mathrm{g}$ of Hgp44-FlaB $(\mathrm{HB})$, or a mixture of $5.1 \mu \mathrm{g}$ of FlaBtFomA plus $8 \mu \mathrm{g}$ of Hgp44-FlaB $(\mathrm{BtA}+\mathrm{HB})$ three times at 2-week intervals as previously described ${ }^{9}$. All animal experimental procedures were conducted in accordance with the guidelines of the Animal Care and Use Committee of the Chonnam National University.

Western blotting analysis

The recombinant proteins or bacterial lysates were separated on an SDS-PAGE gels and transferred onto nitrocellulose membranes (Amersham, 10600004). The anti-tFomA, anti-Hgp44, anti-FlaB, anti- $F$. nucleatum (anti-Fn), or anti- $P$. gingivalis (anti- $\mathrm{Pg}$ ) sera were incubated with the respective membranes for $2 \mathrm{~h}$ at room temperature (RT) to detect corresponding proteins. The horseradish peroxidase (HRP)-conjugated secondary antibody (Dako, P0260) were used to visualize proteins following the manufacture's instruction.

\section{NFkB luciferase reporter assay}

To test whether the FlaB-fusion proteins maintain TLR5-stimulating activities, we determined TLR5-depedent NFKB stimulating activity of the recombinant-fusion proteins as previously described 27. Briefly, $2 \times 10^{5}$ of HEK293T cells/well of culture plate (Corning costar, 3526) were transfected with p3x Flag-hTLR5 (100 ng/well), the pNFkB-luc (100 ng/well), and $50 \mathrm{ng} /$ well of pCMV- $\beta$-Gal using $5 \mu \mathrm{l} /$ well of Effectene (Qiagen, 301427). Twentyfour hours after transfection, the cells were treated with respective LPS-free recombinant proteins ( $100 \mathrm{ng} \mathrm{FlaB}, 126 \mathrm{ng}$ FlaB-tFomA, or $209.5 \mathrm{ng}$ Hgp44-FlaB per well) for 18 hours. The PBS-treated cells were used as a control group. The luciferase activities of the cell lysates were measured by a luminometer (Berthold, Lumat-Plus LB 96V).

Determination of antigen-specific antibody titers

To determine antigen-specific antibody ( $\mathrm{Ab}$ ) titers, the serum and saliva were collected from the immunized mice at two weeks after the final immunization as previously described ${ }^{9}$. The ELISA plates (Corning Laboratories, 3690) were coated by incubation with $1 \mu \mathrm{g} /$ $\mathrm{ml}$ of tFomA or Hgp44 in PBS for 24 hours at $4^{\circ} \mathrm{C}$. The plates were washed with PBST (0.05\% Tween-20 in PBS) and blocked with blocking buffer [0.5\% BSA (Sigma, cat. A2153-50G), 1 mM EDTA (BIONEER, C-9007) in PBST] at RT for 2 hours. The serially diluted sera or saliva were added into the plates and incubated for 2 hours at RT and then the 5 washes were repeated.The HRPconjugated anti-mouse $\lg$ or $\lg A$ antibodies were used as secondary Abs. The signal was developed with $30 \mu \mathrm{l}$ of $3,3^{\prime} 5,5^{\prime}-$ tetramethylbenzidine (TMB) substrate (BD OptEIA, 555214). The reaction was stopped by the addition of $30 \mu \mathrm{l}$ of $1 \mathrm{~N} \mathrm{H}_{2} \mathrm{SO}_{4}$. The optical density was measured with a microplate reader (Molecular Devices Corp., Menlo Park, CA) at $450 \mathrm{~nm}$. The titers were expressed as the reciprocal log2 value of the dilution that yielded 2 -fold higher values of optical density at $450 \mathrm{~nm}$ than the no serum blank well.
Immunostaining and confocal imaging

Fresh cultures of $F$. nucleatum subsp. polymorphum ATCC 10953 or $P$. gingivalis ATCC 33277 were harvested and washed with PBS. One microliter of the bacterial suspension containing $1 \times 10^{8}$ cells was applied to a slide glass and incubated at room temperature until the glass slides were completely dried. Then, the cells on the glass slides were fixed with $4 \%$ paraformaldehyde (T\&l, BPP-9004) for 15 minutes at room temperature. After fixation, the slides were washed three times with PBST (PBS with 0.5\% TWEEN-20) and blocked with $500 \mu \mathrm{l}$ of blocking solution [0.5\% BSA (Sigma, cat. A2153-50G), 1 mM EDTA (BIONEER, C-9007) in PBST] for 60 minutes. The anti-tFomA, anti-BtA, anti-F. nucleatum (anti-Fn), anti-Hgp44, anti-HB, anti- $P$. gingivalis (anti-Pg), or pre-immune sera were incubated with the slides for 1 hour at room temperature (RT) to detect the corresponding FomA and Hgp44 antigens on F. nucleatum and $P$. gingivalis cells, respectively. The glass slides were then washed three times with PBST within 10 minutes of each wash. This wash was followed by incubation with a FITC-labeled anti-mouse IgG secondary antibody (Molecular Probes, Alexa Flour 546) for 1 hour at RT. Confocal images were obtained with a TCS SP5/AOBS/Tandem confocal microscope (Leica Microsystems)

F. nucleatum biofilm formation inhibition assay

The inhibitory effect of anti-sera or anti-saliva on $F$. nucleatuminduced biofilm formation was determined as previously described $^{18}$. The serially diluted anti-sera or anti-saliva in PBS were incubated with freshly prepared $5 \times 10^{9} \mathrm{~F}$. nucleatum subsp. polymorphum ATCC 10953 cells on high-binding 96-well plates (COSTAR, cat\#3590) at RT for 3 hours. The plates were further incubated in anaerobic conditions for 24 hours and then gently washed once with PBS. The wells were stained with $25 \mu$ of $0.3 \%$ crystal violet for 15 minutes and gently washed with PBS. The stained biofilm was extracted with $100 \mu \mathrm{l}$ of ethanol, and the optical density was measured with a microplate reader (Molecular Devices Corp., Menlo Park, CA) at $595 \mathrm{~nm}$.

Biofilm staining and confocal imaging

Freshly prepared $1 \times 10^{9} \mathrm{~F}$. nucleatum subsp. polymorphum ATCC 10953 cells were applied on cell culture slides (SPL cat 30504) and the slides were placed at $37^{\circ} \mathrm{C}$ under an anaerobic conditions ( $85 \%$ $\mathrm{N} 2,10 \% \mathrm{H} 2$, and $5 \% \mathrm{CO} 2$ ) overnight and gently washed once with PBS. The biofilm was stained with DAPI and then mounted with ProLong Gold antifade reagent (Life Technologies, P36935). Confocal images were obtained with an LSM510 confocal microscope.

\section{Hemagglutination inhibition assay}

The $P$. gingivalis-induced HIA was performed as previously described $^{9}$. The serially diluted anti-sera or anti-saliva with PBS were incubated with $1 \times 10^{8} \mathrm{P}$. gingivalis ATCC 33277 cells at $37^{\circ} \mathrm{C}$ for 1 hour. Then, a mouse red blood cell (RBC) suspension was added to the plate at $1 \%(\mathrm{v} / \mathrm{v})$ of the finial concentration. The plate was incubated at $37^{\circ} \mathrm{C}$ for 3 hours to observe the hemagglutination reaction.

Adhesion and cytotoxicity assay

To determine the effect of anti-sera on the $P$. gingivalis-host cell interactions, we performed adhesion and cytotoxicity assays as previously described ${ }^{43}$. HGFs grown overnight on chamber slide (SPL Life Science, cat. 30124) were washed twice with serum/ antibiotic-free DMEM and incubated with purified IgG from 1/16fold diluted sera. The IgGs were purified from the appropriate sera using the lgG purification kit (Thermo Scientific, cat. 45206) following the manufacturer's instruction. Then, the HGFs were infected with $P$. gingivalis ATCC 33277 at an MOI of 100 . To analyze the adhesion of $P$. gingivalis on HGFs, 90 minutes after the bacterial infection the chamber slides were thoroughly washed three times with pre-warmed DMEM and stained with Giemsa 
solution (Merck, cat. 940383307). Images were acquired with light microscope (Nikon, S/N. KC300211191) under 1,000X magnification. Since P. gingivalis appeared to form microcolonies adhered to the host's surface and could not be counted as single bacterium numbers per host cell, we calculated the bacterial adhesion as the number of microcolonies per field on 1000X magnification. To test the $P$. gingivalis-mediated cytotoxicity, we measured LDH release from the HGFs using the CytoTox96 nonradioactive cytotoxic assay kit (Promega G1780) in accordance with the manufacturer's protocol.

\section{Bacterial co-aggregation assay}

To test the effect of anti-sera on the $P$. gingivalis-Treponema denticola interaction, we performed bacterial co-aggregation assays as previously described ${ }^{35}$. Briefly, fresh $P$. gingivalis ATCC 33277 and T. denticola ATCC 35405 cultures were harvested and washed twice with co-aggregation buffer $(10 \mathrm{mM}$ Tris- $\mathrm{HCl}, 150$ $\mathrm{mM} \mathrm{NaCl}, 1 \mathrm{mM} \mathrm{CaCl} 2,1 \mathrm{mM} \mathrm{MgCl}_{2}$ and $0.02 \% \mathrm{NaN}_{3}, \mathrm{pH} 8.0$ ), the final pellets were resuspended in co-aggregation buffer, and the $\mathrm{OD}_{600}$ of the cell suspension was adjusted to 1.0. Then, $P$. gingivalis and $T$. denticola were mixed in 2:1 ratio and transferred into cuvettes. Purified-lgG from various sera were subsequently added into each cuvette. Purified-lgG from anti-Pg and pre-immune sera were used as controls. To evaluate co-aggregation, cuvettes were incubated at room temperature for 30,60, 90, and 120 minutes. The co-aggregation of $P$. gingivalis and $T$. denticola was monitored by measuring the $\mathrm{OD}_{600}$ of the cell suspension for 2 hours at 30 minute intervals.

\section{Flow cytometry}

Immune cells isolated from the cervical lymph nodes or spleens were incubated with anti-mouse CD16/32 antibody (Biolegend, San Diego, CA, USA). To identify the GC B cells, Tfh cells and plasma cells, the following antibodies were purchased from Biolegend: anti-GL7-FITC, anti-CD19-APC, anti-CD4-FITC, antiCXCR5-APC, anti-PD1-PE, anti-B220-FITC, and anti-CD138-PE. After staining, the cells were analyzed by an Accuri C6 Flow Cytometer System (BD Bioscience).

\section{Quantitative RT-PCR}

Gene expression levels were analyzed by qRT-PCR analysis. Total RNA was isolated from samples using TRlzol (Invitrogen, USA) or RNeasy kits (Qiagen). The total RNA was reverse transcribed using the SuperScript ${ }^{\circledR}$ II Reverse Trasncriptase (Invitrogen, USA) and an oligo-dT primer. Quantitative PCR was performed on a RoterGene $^{\mathrm{TM}} 6000$ (Corbett Life Science, Mortlake, Sydney, Australia) using the SensiMix SYBR $^{\circledR}$ Green mixture (Quantace, Germany). The qRT-PCR data are generated from all the $\mathrm{Ct}$ values which were normalized against $\beta$-actin levels and normalized fold-change were calculated following the $2^{-\Delta \Delta} \mathrm{Ct}$ method. The primers used in the experiments are listed in Supplementary Table 3.

Bacterial challenge with live $F$. nucleatum and $P$. gingivalis To test whether periodontal vaccines elicit protective immune responses in a periodontal disease induced by $F$. nucleatum and $P$. gingivalis mixed infection, we performed challenge assay. Briefly, 2 weeks after the last vaccination, the mice were orally infected with $1 \times 10^{9} \mathrm{CFU}$ of $F$. nucleatum subsp. polymorphum ATCC 10953 and $1 \times 10^{9}$ CFU of $P$. gingivalis ATCC 33277 in $2 \%$ of carboxymethylcellulose (Sigma, IGEPAL ${ }^{\circledR}$ CA-630) 3 times with a 2-day interval between treatments (Fig. 7A). To avoid of the competition of the mixed bacteria ( $F$. nucleatum and $P$. gingivalis) with oral microbiome in the colonization step, the vaccinated mice were pretreated with $2 \mathrm{mg} / \mathrm{ml}$ of sulfamethoxazole and $0.4 \mathrm{mg} / \mathrm{ml}$ of trimethoprim (Samil Pharm, Septrin Syrup) for 3 days and subjected to an antibiotic-free period of 3 more days as previously described ${ }^{9}$.
Micro-computed tomography (micro-CT) analysis

Micro-CT analysis was performed as previously described ${ }^{9}$. Briefly, the right sides of the maxillae were sampled and fixed in $10 \%$ formalin. The Skyscan 1172 CT-system (Skyscan, Aartselaar, Belgium) with an x-ray electromagnetic source at $50 \mathrm{kV}$ and 201 $\mathrm{mA}$ with a $0.5-\mathrm{mm}$ aluminum filter was used to scan the micro-CT images which produced the images with a pixel size of $15 \mathrm{~mm}$ at every $0.7^{\circ}$ over an angular range of $180^{\circ}$. Then the scanned images were reconstructed to generate three-dimensional microstructures (0 to 8,000 in Hounsfield units) using an image reconstruction software (version 1.6.2.0, Skyscan). The bone volume fraction (bone volume/tissue volume; BV/TV) in the region of interest (ROI) was determined using the CT Analyzer program (version 1.10.0.5, Skyscan) as previously described. Three-dimensional surface rendering images were obtained with the Mimics imaging program (version 14.0, Materialise). The ROI length extended from the most distal aspect of the $M 2$ root to the most mesial aspect of the $M 1$ root. The width extended from the most buccal aspect to the most palatal (lingual) part of the M1 or M2 root, including the alveolar bone, and the height extended from the most apical aspect of the M1 or M2 crown to the most apical aspect of the M1 or $M 2$ root. The linear distance of the CEJ to the $A B C$ in maxillae M1 and M2 was determined using the Mimics software 14.0 (Materialise).

\section{Statistical analysis}

The results are expressed as the mean \pm SEM unless otherwise noted. Student's t-test was used to compare two groups. All experiments were repeated more than three times, and the results from representative experiments are shown.

\section{ACKNOWLEDGEMENTS}

S.E.L. was supported by an NRF grant from the MSIP [NRF-2016R1A2B4009611] and by a COMPA grant from the MSIP (2017K000360) of the Republic of Korea. J.H.R. was supported by a grant from the National Program for Cancer Control, Ministry of Health \& Welfare of the Republic of Korea HA17C0038 (1720120) and by the Bio \& Medical Technology Development Program of the NRF funded by the Korean government, MSIP (NRF-2017M3A9E2056372). H.S.N. was supported by an NRF grant from the MSIP [NRF-2017M3A9B6062021].

\section{AUTHOR CONTRIBUTIONS}

Substantial contributions to conception and design of this study: L.S.E., R.J.H.; Acquisition of data: P.S., H.S.H., L.H.H., L.Y.S., S.S., A.S.J., N.H.S.; Analysis and interpretation of data: P.S., H.S.H., N.H.S., Ryu.J.H., A.S.J., K.J.K., K.I.C., K.J.H., L.S.E., R.J. H.; Drafting the article: P.S., H.S.H., L.S.E., R.J.H.; Revising it critically for important intellectual content: P.S., L.S.E., R.J.H.; Final approval of the version to be published: L. S.E., R.J.H.

\section{ADDITIONAL INFORMATION}

The online version of this article (https://doi.org/10.1038/s41385-018-0104-6) contains supplementary material, which is available to authorized users.

Competing interests: The authors declare no competing interests.

\section{REFERENCES}

1. Dorfer, C., Benz, C., Aida, J. \& Campard, G. The relationship of oral health with general health and NCDs: a brief review. Int. Dent. J. 67(Suppl 2), 14-18 (2017).

2. Hajishengallis, G. Periodontitis: from microbial immune subversion to systemic inflammation. Nat. Rev. Immunol. 15, 30-44 (2015).

3. Hajishengallis, G. \& Lamont, R. J. Beyond the red complex and into more complexity: the polymicrobial synergy and dysbiosis (PSD) model of periodontal disease etiology. Mol. Oral. Microbiol. 27, 409-419 (2012).

4. Hasan, A. \& Palmer, R. M. A clinical guide to periodontology: pathology of periodontal disease. Br. Dent. J. 216, 457-461 (2014). 
5. Shi, B. et al. Dynamic changes in the subgingival microbiome and their potential for diagnosis and prognosis of periodontitis. mBio 6, e01926-01914 (2015).

6. Hong, B. Y. et al. Microbiome profiles in periodontitis in relation to host and disease characteristics. PLOS ONE 10, e0127077 (2015).

7. Diaz, P. I., Hoare, A. \& Hong, B. Y. Subgingival microbiome shifts and community dynamics in periodontal diseases. J. Calif. Dent. Assoc. 44, 421-435 (2016).

8. Stone, V. N. \& Xu, P. Targeted antimicrobial therapy in the microbiome era. $\mathrm{Mol}$ Oral Microbiol. 32:446-454 (2017).

9. Puth, S. et al. Mucosal immunization with a flagellin-adjuvanted Hgp44 vaccine enhances protective immune responses in a murine Porphyromonas gingivalis infection model. Hum. Vaccin. Immunother. 13, 2794-2803 (2017).

10. Grover, V., Kapoor, A., Malhotra, R. \& Kaur, G. Porphyromonas gingivalis antigenic determinants-potential targets for the vaccine development against periodontitis. Infect. Disord. Drug. Targets 14, 1-13 (2014).

11. Reynolds, E. C. et al. Prospects for treatment of Porphyromonas gingivalismediated disease - immune-based therapy. J. Oral. Microbiol. 7, 29125 (2015).

12. Hajishengallis, G. Immunomicrobial pathogenesis of periodontitis: keystones, pathobionts, and host response. Trends Immunol. 35, 3-11 (2014)

13. Socransky, S. S. \& Haffajee, A. D. Evidence of bacterial etiology: a historical perspective. Periodontol. 2000. 5, 7-25 (1994).

14. Olsen, I., Lambris, J. D. \& Hajishengallis, G. Porphyromonas gingivalis disturbs host-commensal homeostasis by changing complement function. J. Oral. Microbiol. 9, 1340085 (2017).

15. Aruni, A. W., Dou, Y., Mishra, A. \& Fletcher, H. M. The biofilm community-rebels with a cause. Curr. Oral. Health Rep. 2, 48-56 (2015).

16. Bakaletz, L. O. Developing animal models for polymicrobial diseases. Nat. Rev. Microbiol. 2, 552-568 (2004).

17. Diaz, P. I., Zilm, P. S. \& Rogers, A. H. Fusobacterium nucleatum supports the growth of Porphyromonas gingivalis in oxygenated and carbon-dioxide-depleted environments. Microbiology 148(Pt 2), 467-472 (2002).

18. Nakagaki, $H$. et al. Fusobacterium nucleatum envelope protein FomA is immunogenic and binds to the salivary statherin-derived peptide. Infect. Immun. $\mathbf{7 8}$ 1185-1192 (2010).

19. Kleinschmidt, J. H. Folding kinetics of the outer membrane proteins OmpA and FomA into phospholipid bilayers. Chem. Phys. Lipids 141, 30-47 (2006).

20. Jeong, K. et al. Development of a novel subunit vaccine targeting fusobacterium nucleatum FomA porin based on in silico analysis. Int. J. Oral. Biol. 42, 63-70 (2017).

21. Park, S. N. et al. Draft Genome Sequence of Fusobacterium nucleatum subsp. fusiforme ATCC 51190 T. J Bacteriol. 194, 5445-5446 (2012).

22. Moutsopoulos, N. M. \& Konkel, J. E. Tissue-specific immunity at the oral mucosal barrier. Trends Immunol. 39:276-287 (2018).

23. Chen, K. \& Cerutti, A. Vaccination strategies to promote mucosal antibody responses. Immunity 33, 479-491 (2010).

24. Rhee, J. H., Lee, S. E. \& Kim, S. Y. Mucosal vaccine adjuvants update. Clin. Exp. Vaccin. Res. 1, 50-63 (2012).

25. Suzuki, T. et al. Relationship of the quaternary structure of human secretory $\lg A$ to neutralization of influenza virus. Proc. Natl Acad. Sci. USA 112, 7809-7814 (2015).

26. Corthesy, B. Multi-faceted functions of secretory IgA at mucosal surfaces. Front. Immunol. 4, 185 (2013).

27. Lee, S. E. et al. A bacterial flagellin, Vibrio vulnificus FlaB, has a strong mucosal adjuvant activity to induce protective immunity. Infect. Immun. 74, 694-702 (2006).

28. Lim, J. S. et al. Flagellin-dependent TLR5/caveolin-1 as a promising immune activator in immunosenescence. Aging Cell 14, 907-915 (2015).

29. Nguyen, C. T., Kim, S. Y., Kim, M. S., Lee, S. E. \& Rhee, J. H. Intranasal immunization with recombinant PspA fused with a flagellin enhances cross-protective immunity against Streptococcus pneumoniae infection in mice. Vaccine 29, 5731-5739 (2011).

30. Rana, A. \& Akhter, Y. A multi-subunit based, thermodynamically stable model vaccine using combined immunoinformatics and protein structure based approach. Immunobiology 221, 544-557 (2016).
31. Muramatsu, M. et al. Class switch recombination and hypermutation require activation-induced cytidine deaminase (AID), a potential RNA editing enzyme. Cell 102, 553-563 (2000).

32. Kim, H. S. et al. Application of rpoB and zinc protease gene for use in molecular discrimination of Fusobacterium nucleatum subspecies. J. Clin. Microbiol. 48, 545-553 (2010)

33. Signat, B., Roques, C., Poulet, P. \& Duffaut, D. Fusobacterium nucleatum in periodontal health and disease. Curr. Issues Mol. Biol. 13, 25-36 (2011).

34. Pocanschi, C. L. et al. The major outer membrane protein of Fusobacterium nucleatum (FomA) folds and inserts into lipid bilayers via parallel folding pathways. J. Mol. Biol. 355, 548-561 (2006).

35. Ito, R., Ishihara, K., Shoji, M., Nakayama, K. \& Okuda, K. Hemagglutinin/Adhesin domains of Porphyromonas gingivalis play key roles in coaggregation with Treponema denticola. Fems. Immunol. Med. Microbiol. 60, 251-260 (2010).

36. Khan, S. A., Kong, E. F., Meiller, T. F. \& Jabra-Rizk, M. A. Periodontal diseases: bug induced, host promoted. PLoS Pathog. 11, e1004952 (2015).

37. Lamont, R. J. \& Hajishengallis, G. Polymicrobial synergy and dysbiosis in inflammatory disease. Trends Mol. Med. 21, 172-183 (2015).

38. Sintim, H. O. \& Gursoy, U. K. Biofilms as "connectors" for oral and systems medicine: a new opportunity for biomarkers, molecular targets, and bacterial eradication. OMICS 20, 3-11 (2016).

39. Kolenbrander, P. E., Palmer, R. J. Jr, Periasamy, S. \& Jakubovics, N. S. Oral multispecies biofilm development and the key role of cell-cell distance. Nat. Rev. Microbiol. 8, 471-480 (2010).

40. Han, Y. W. Fusobacterium nucleatum: a commensal-turned pathogen. Curr. Opin. Microbiol. 23, 141-147 (2015).

41. Socransky, S. S. et al. Microbial complexes in subgingival plaque. J. Clin. Periodontol. 25, 134-144 (1998).

42. Naito, M. et al. Porphyromonas gingivalis-induced platelet aggregation in plasma depends on Hgp44 adhesin but not Rgp proteinase. Mol. Microbiol. 59, 152-167 (2006).

43. Kim, S. Y. et al. Contribution of six flagellin genes to the flagellum biogenesis of Vibrio vulnificus and in vivo invasion. Infect. Immun. 82, 29-42 (2014).

44. Lee, S. E. et al. Flagellin is a strong vaginal adjuvant of a therapeutic vaccine for genital cancer. Oncoimmunology 5, e1081328 (2016).

45. Boyaka, P. N. \& Inducing Mucosal IgA: a challenge for vaccine adjuvants and delivery systems. J. Immunol. 199, 9-16 (2017).

46. Brandtzaeg, P. Secretory lgA: designed for anti-microbial defense. Front. Immunol. 4, 222 (2013).

47. Cao, A. T. et al. Interleukin (IL)-21 promotes intestinal IgA response to microbiota. Mucosal Immunol. 8, 1072-1082 (2015).

48. Cyster, J. G. B cell follicles and antigen encounters of the third kind. Nat. Immunol. 11, 989-996 (2010).

49. Hong, S. H. et al. Intranasal administration of a flagellin-adjuvanted inactivated influenza vaccine enhances mucosal immune responses to protect mice against lethal infection. Vaccine 30, 466-474 (2012).

50. Gil-Montoya, J. A., de Mello, A. L., Barrios, R., Gonzalez-Moles, M. A. \& Bravo, M. Oral health in the elderly patient and its impact on general well-being: a nonsystematic review. Clin. Interv. Aging 10, 461-467 (2015).

Open Access This article is licensed under a Creative Commons Attribution 4.0 International License, which permits use, sharing, adaptation, distribution and reproduction in any medium or format, as long as you give appropriate credit to the original author(s) and the source, provide a link to the Creative Commons license, and indicate if changes were made. The images or other third party material in this article are included in the article's Creative Commons license, unless indicated otherwise in a credit line to the material. If material is not included in the article's Creative Commons license and your intended use is not permitted by statutory regulation or exceeds the permitted use, you will need to obtain permission directly from the copyright holder. To view a copy of this license, visit http://creativecommons. org/licenses/by/4.0/.

(c) The Author(s) 2018 